\title{
II. Die Anfänge des deutsch-israelischen Dialogs
}

In den ersten Nachkriegsjahren traten der Jischuv, die jüdische Gemeinschaft Palästinas, und nach 1948, die jüdischen Bürger Israels, Deutschland und Deutschen mit offener Feindseligkeit gegenüber. Ein amerikanischer Diplomat in Tel Aviv berichtete seinen Vorgesetzten über einen "fast pathologischen $\mathrm{Haß}^{3}$ “ gegenüber allem Deutschen in breiten Teilen der israelischen Öffentlichkeit. ${ }^{1}$ Folgende scharfe Äußerungen veranschaulichen diese Stimmung: „Wir müssen unsere Kinder und Enkel schon von früh auf $\mathrm{Haß}$ gegen Deutsche einschärfen. Die Rache muß und wird kommen, wenn wir stark sind", schrieb die Journalistin Orah Shem-Tow 1949 in der israelischen Tageszeitung Yedioth Achronoth. ${ }^{2}$ Der Historiker und Arzt Dr. Mark Dworecki, dessen Familie im Holocaust umgekommen war, sagte während der Schilumimdebatte im Präsidium der Regierungspartei Mapai Ende 1951 in Anlehnung an das biblische Leitwort „Auge um Auge, Zahn um Zahn“: „Auf die Frage, was ich von Deutschland verlange, würde ich antworten: Mutter um Mutter, Vater um Vater und Kind um Kind. Meine Seele wird nicht eher zur Ruhe kommen, als daß ich sechs Millionen Deutsche für die sechs Millionen Juden sterben sehe." Im selben Forum äußerte die Mapai-Politikerin Golda Meyerson (die spätere Ministerpräsidentin Golda Meir): „Mein Standpunkt scheint mir realistisch: Für mich ist jeder Deutsche als solcher ein Nazi. “3

Die Emotionen in der israelischen Öffentlichkeit gegenüber Deutschland gewannen zunehmend politische Bedeutung. Vor allem von den Holocaust-Überlebenden unter den Einwanderern getragen, wurde der $\mathrm{Haß}$ auch anderen Teilen der Bevölkerung, unabhängig von ihrer Herkunft, weitervermittelt, auch solchen, die vom Holocaust nicht direkt betroffen gewesen waren.

Andererseits gab es jüdische Bevölkerungsteile in Israel, die dem Kollektivurteil über Deutsche kritisch gegenüberstanden und diese Haltung als zu extrem ablehnten. $\mathrm{Zu}$ ihnen zählten zahlreiche Juden deutscher Herkunft, Einwanderer aus angelsächsischen Ländern, Teile des orientalischen Judentums, Linke und Vertreter der alten liberalen Tradition. Gemäßigte israelische Persönlichkeiten angelsächsischer Herkunft leisteten wenige Jahre später einen wesentlichen Beitrag zur politischen Annäherung zwischen Israel und der Bundesrepublik Deutschland. Aber auch vor dem Krieg emigrierte deutsche Juden zeigten mehr Verständnis für ihre ehemalige Heimat. Einige führende Vertreter der Jewish Agency for Palestine (JAFP), die sich für eine Aufnahme des Dialogs zwischen der Bundesrepublik Deutschland und Israel einsetzten, waren deutscher Herkunft. Jüdische Intellek-

1 Richard Ford an das Außenministerium vom 27. 10. 1950, USNA, RG-84, Consular Post Tel Aviv, 1950-52.

2 Segev, 1949, S. 289.

3 Protokoll der Sitzung des MAPAI-Zentralkomittees am 13. 12. 1951, LPA, Protokolle, Bd. 3, Nr. 23/51. 
tuelle aus dem deutschen Kulturkreis, darunter Martin Buber und Ernst Simon, betonten die wichtige Rolle der Juden bei der Heranbildung einer neuen deutschen Generation und trugen selbst zur Prägung von vorurteilsfreieren Ansichten und Haltungen hinsichtlich Deutschlands in Israel bei. Jüdische Einwanderer aus orientalischen Ländern, etwa aus Nordafrika, dem Libanon und dem Irak, waren entweder der Verfolgung durch die Nationalsozialisten selbst oder durch ihre Verbündeten ausgesetzt gewesen und solidarisierten sich deshalb mit ihren Mitbürgern europäischer Herkunft. Einwanderer aus anderen, vom Holocaust nicht direkt betroffenen Ländern, konnten das Erlittene und den Haß zumindest emotional und intellektuell nachvollziehen, ihre Gefühle bzw. ihre Abneigung gegenüber Deutschland waren aber zwangsläufig weniger intensiv als die jener Einwanderer, deren Angehörige von den Nationalsozialisten ermordet worden waren.

Den Ton des antideutschen Ressentiments in Israel bestimmten aber eindeutig die Holocaust-Überlebenden. Dieses wurde oft noch von anderen Gruppen verstärkt, darunter selbst von Teilen der Bevölkerung, die vom Holocaust nicht persönlich betroffen waren. Die Stimmen jener, die Kollektivurteile über Deutsche ablehnten, waren dagegen kaum zu vernehmen. Sogar linke Parteien, bekannt für ihre internationalistische Tradition, nahmen hinsichtlich Deutschland eher einen politisch-emotionalen als einen ihrer Ideologie angemessenen Standpunkt ein. Der Haß gegenüber Deutschland war besonders eklatant bei der sozialdemokratischen Mapai, bei der links von ihr stehenden Mapam sowie bei der Maki, der kommunistischen Partei Israels. Mapam und Maki legten Wert auf eine Unterscheidung zwischen der Bundesrepublik und der Deutschen Demokratischen Republik. Kam es jedoch zu einer emotionalen Debatte, was oft der Fall war, fielen auch diese Parteien auf die verallgemeinernden antideutschen Schemata zurück. Dagegen wurden Deutsche mit anti-nationalsozialistischen und projüdischem Hintergrund von Verfechtern humanistischer Werte in Israel mit offenen Armen empfangen. Sie weigerten sich, einen Gast abzuweisen, nur weil er Deutscher war. So wurde etwa dem Heidelberger Dekan Heinrich Maas in Israel ein sehr freundschaftlicher Empfang zuteil, wenn auch sein Besuch unter Ausschluß der Öffentlichkeit stattfinden mußte. ${ }^{4}$ Zahlreiche Mitglieder rechter Parteien, vor allem der extremistischen Cherut-Partei, lehnten jeden Kontakt mit Deutschen, auch wenn diese unbelastet waren, $a b$.

\section{Boykott und Bann}

Die antideutsche Tendenz in Israel äußerte sich besonders durch einen inoffiziellen Bann über Deutschland und allem Deutschen. Typisch dafür war etwa ein Beschluß der JAFP gegen Wirtschaftsbeziehungen mit Deutschland. Die Exekutive dieser Organisation trug dabei der - wirtschaftlichen - Realität insofern Rechnung, als sie einschränkend erklärte: „Die Exekutive hat nichts gegen die Einfuhr des Eigentums von Einwanderern (Maschinen etc.). Sie ist jedoch gegen die Wie-

4 Die Neue Zeitung vom 8. 3. 1950. 
deraufnahme von Handelsbeziehungen mit Deutschland. “5 Der führende MapaiWirtschaftsexperte Aharon Remez sagte auf einer Veranstaltung: „Es ist Zeit, über einen langjährigen Bann gegen Deutschland und über einen Boykott deutscher Produkte zu sprechen." ${ }^{\text {Im Januar }} 1950$ untersagte die israelische Regierung den Handel mit Deutschland durch Regierungsbeschluß.? Der anschließend mehrmals bekräftigte Boykott mußte in der Folge jedoch aufgegeben werden. Er war schlicht nicht durchsetzbar, die Kräfte der Wirtschaft waren offensichtlich stärker. Doch auch die israelische Außenpolitik gegenüber Deutschland war nicht frei von Widersprüchen. Sie schwankte zwischen antideutschen Erklärungen und der Pflege von Außenbeziehungen mit deutschen Partnern. Sowohl die Bundesrepublik als auch die DDR waren für das israelische Außenministerium zunächst ein Anathema. Sechs Monate nach seiner Gründung eröffnete der Staat Israel ein Konsulat in München, das ausschließlich bei den Besatzungsmächten akkreditiert war. Den Mitarbeitern des Konsulats war es strikt untersagt, irgendwelche Kontakte mit deutschen Behörden zu knüpfen. Diese Anweisung war zwar nicht einzuhalten, das Konsulat tat dennoch sein Bestes. ${ }^{8}$ Israelische Diplomaten wurden angewiesen, ihre deutschen Partner zu boykottieren, ohne Unterschied zwischen West und Ost. Doch auch in diesem Fall erwies sich die Realität stärker als die offiziellen Direktiven. Es gelang den Israelis nicht, sich völlig abzugrenzen. ${ }^{9}$

Das israelische Außenministerium und das israelische Einwanderungsministerium versuchten sodann, den gegenseitigen Besucherstrom zwischen Deutschland und Israel zu unterbinden. Anfang 1950 wurde der israelische Paßvermerk „für alle Länder gültig" mit dem Stempel „außer für Deutschland“ ergänzt. Doch wie zuvor hatte diese Maßnahme nur auf dem Papier Bestand. Israelische Staatsbürger konnten sich deutsche Visa leicht in deutschen Konsulaten in Europa beschaffen. Der diskriminierende Paßstempel wurde 1956 wieder abgeschafft ${ }^{10}$ - auf Veranlassung des israelischen Konsuls Eliahu Kurt Livneh ${ }^{11}$-, zum Bedauern der bundesdeutschen Behörden, die nach Wegen suchten, den als zu stark empfundenen Einreisestrom israelischer Staatsbürger einzuschränken. Einfacher war es, die Einreise Deutscher nach Israel zu verhindern, obwohl auch das nicht vollständig gelingen konnte. Soweit bekannt, fand der erste offizielle Besuch eines deutschen Staatsbürgers auf israelischem Hoheitsgebiet unter den Auspizien des Vatikans statt. Es handelte sich um den Besuch eines Emissärs des Vereins vom Heiligen Lande, der im Auftrag reiste, das Eigentum der katholischen Kirche zu inspizieren. Kreisdekan Hermann Maas war damit der erste offizielle deutsche Gast in

5 Protokoll der JAFP-Exekutivausschuß-Sitzung in Jerusalem am 8. 7. 1947, BGA, Protocol Files.

6 Ebd.

7 Der Rechtsberater an den Außenminister vom Dezember 1949, ISA, 2416/15a; KNESSETPROTOKOLLe [hebr.], 108. Sitzung am 23. 1. 1950.

8 JELINEK, Like an Oasis, S. 88-93.

9 JELINEK/WOLFFSOHN, Berührungsängste, S. 282-288.

10 Zusammenfassung einer Besprechung über Angelegenheiten betr. Deutschland vom Dezember 1955, ISA, Chet/4321a; Abteilung Westeuropa an das Ministerbüro vom 4. 1. 1956; der Leiter der Konsularabteilung an den Generaldirektor vom 8.1. 1956, ISA, 2413/3b.

11 Livneh an den Generaldirektor vom 8. 1. 1956, ISA, 2413/36. 
Israel und Rudolf Küstermeier von der Hamburger Zeitung Die Welt der erste deutsche Journalist, der ins Land einreisen durfte.

Die Schiffahrt war vom Deutschland-Boykott zumindest teilweise ausgenommen: Israelische Schiffe frequentierten deutsche Häfen uneingeschränkt, während die israelischen Territorialgewässer für deutsche Schiffe gesperrt blieben. Noch im Juli 1951 war der nationalen Fluggesellschaft El Al die Benutzung deutscher Flughäfen untersagt. Israelische Hochschulen und wissenschaftliche Institute litten besonders unter dem Boykott, da er den Import wissenschaftlicher Instrumente und wissenschaftlicher Literatur sowie den Aufbau akademischer Austauschprogramme behinderte. Deutsche Kulturerzeugnisse waren vom öffentlichen Leben in Israel fast völlig verbannt, doch auch in diesem Fall war die Realität stärker als der erklärte Boykott.

Ein Bereich, in dem die Einhaltung des Boykotts streng beachtet wurde, war die Presse. Der in Deutschland geborene Herausgeber der angesehenen israelischen Tageszeitung Haaretz, Gershom Schocken, fiel durch eine besonders radikale Haltung auf. In den Leitartikeln der Ausgaben vom 2. und 4. September 1949 rief er zu einem totalem Boykott Deutschlands und zu einem Abbruch der Handelsbeziehungen mit Deutschland auf. Von dieser Haltung abweichende Standpunkte wurden in seiner Zeitung nicht geduldet.

Versuche jüdischer Großhändler außerhalb Israels, einen weltweiten Boykott deutscher Waren durchzusetzen, waren - aus praktischen Gründen - ebenso zum Scheitern verurteilt, wie die Proklamation des Boykotts gegenüber Deutschland durch den Jüdischen Weltkongreß im Herbst 1950. Kaum erfolgreicher war der Plan der JAFP, bis Ende 1949 alle sich in Deutschland aufhaltenden Juden zur Ausreise zu bewegen und sämtliche öffentlichen jüdischen Institutionen in Deutschland zu schließen. ${ }^{12}$ Der Bann und die verschiedenen Boykotte symbolisierten das tiefe Ressentiment der Israelis (und der Juden) gegen alles, was irgendwie mit Deutschland zusammenhing.

In der Knesset, im israelischen Parlament, kam die Abneigung gegen die Erben des Dritten Reichs in verschiedenen Gesetzen zum Ausdruck. 1949 verabschiedete die erste Knesset ein Gesetz über das deutsche Eigentum (auf israelischem Territorium), das in der Bundesrepublik Deutschland große Empörung auslöste, da es dort - zu Unrecht - als Beschlagnahme deutschen Besitzes im Heiligen Land interpretiert wurde. In Wirklichkeit wurde der deutsche Besitz bloß zum Pfand für spätere Reparationen erklärt. ${ }^{13}$ Auch die Verabschiedung des Gesetzes über die Verfolgung der Nationalsozialisten und deren Helfer sowie die internationale Konvention zur Verhütung und Bestrafung von Völkermord waren in der ersten Knesset von langen Debatten begleitet. Zieht man außerdem noch die Knessetdebatten über den Handel mit Deutschland, über Entschädigungsforderungen sowie über einige weitere mit Deutschland zusammenhängende Themen in Betracht, ergibt sich das Bild einer massiven Beteiligung der israelischen Legislative an der

12 Yachil, Köln, an Levavi, Jerusalem, vom 16. 11. 1953, ISA, 2519/4; SHAFIR, Der Jüdische Weltkongreß, S. 218-219.

13 Zwischen Moral und RealPolitik, Dokument Nr. 3, S. 134; Protokoll der Regierungssitzung Nr. 310/10 am 1. 11. 1949, ISA, 7263. 
Distanzierung Israels und der Juden gegenüber Deutschland. ${ }^{14}$ Kurzum: Bann, Boykott und Distanz charakterisierten die erste Periode der deutsch-israelischjüdischen Beziehungen in der Nachkriegszeit.

Eine besonders bedeutsame und deshalb in der jüdischen Öffentlichkeit und in Israel häufig diskutierte Frage betraf das Verhältnis zwischen den Deutschen als Kollektiv und den NS-Verbrechen. Sie wurde sowohl in weiterer Perspektive, d.h. hinsichtlich der Kriegsschuld der Nationalsozialisten und der NS-Verbrechen gegen die Menschlichkeit, als auch spezifisch im Hinblick auf die „Endlösung der Judenfrage" behandelt. Die Nürnberger Prozesse - ein präzedenzloses Ereignis in der Menschheitsgeschichte - bezweckten mindestens zum Teil eine Auseinandersetzung mit dieser Frage gemäß der weiteren Perspektive. Die beispiellosen Prozesse wurden aber auch zum Präzedenzfall für die später erfolgenden deutschen Reparationen an das jüdische Volk, selbst ein Phänomen ohne Beispiel.

$\mathrm{Zu}$ Verallgemeinerungen neigend, machten Teile der jüdischen Öffentlichkeit die Deutschen generell für die NS-Verbrechen verantwortlich. Dementsprechend undifferenziert manifestierten sich die antideutschen Gefühle zunächst in den DP-Camps, danach wandelten sie sich in Israel zu einer sozialen Kraft und fanden schließlich Widerhall in anderen jüdischen Gemeinschaften rund um den Erdball. Solche Pauschalurteile wurden später von Intellektuellen wie auch von Politikern, sei es aus moralischen oder aus politisch-pragmatischen Gründen, abgelehnt. Dabei wurde versucht, zwischen „Schuldigen“ und „Unschuldigen“ zu differenzieren und die Täter von den Mitbelasteten oder den Mitläufern zu scheiden. ${ }^{15}$ Doch die Kollektivschuldfrage beschäftigte nicht nur die jüdische Seite. Sie lastete schwerer noch auf den politischen, intellektuellen, religiösen und sozialen Eliten der Bundesrepublik Deutschland. In zahlreichen politischen, ethischen und intellektuellen Debatten wurde dort versucht, zwischen Schuld und Scham, Kollaborateuren und Mitläufern, Mitwisserschaft und Nichtwissen, Vorbild und Nachahmung zu unterscheiden. ${ }^{16}$ Der merkwürdige historische Zufall hatte das jüdische und das deutsche Schicksal untrennbar miteinander verbunden. Dem Holocaust war ein metamorphischer Einfluß auf das Selbstverständnis sowohl der Täter als auch der Opfer beschieden, der kurz nach dem Zusammenbruch des Dritten Reiches deutlich zum Ausdruck kam und sich als treibende Kraft nach der Gründung des jüdischen Staates und der beiden neuen deutschen Staaten erwies.

14 Protokoll der Regierungssitzung Nr. 310/11 am 29. 11. 1949, Protokoll der Regierungssitzung Nr. 319/12 vom 5. 12. 1949, ISA, 7263/4; KNESSET-PrOTOKOLLE [hebr.], 90. Sitzung am 29.11. 1949, S. 111, 108. Sitzung am 23. 1. 1950, S. 120, 131. Sitzung am 27.1. 1950, S. 147-162; HAARETZ vom 24. 1., 17.6. und 2.8. 1950.

$15 \mathrm{Zu}$ den Debatten in Israel über Deutschland und die Deutschen: Barzel, The Attitude of Jews; BARZEL, Dignity, Hatred and Memory; BARZEL, Hatvia'a b'yishuv l'haanshatah. Zu den Debatten unter den Holocaust-Überlebenden und den DP's in Deutschland: Bergen Belsen Archives in Honor of Yosef Rozensaft, No. 0-70, Yad Vashem Archives, Jerusalem.

$16 \mathrm{Zu}$ den Debatten in Deutschland bis 1950: JochManN, Gesellschaftskrise und Judenfeindschaft; SCHWIERIGES ERBE; WOLFFSOHN, Ewige Schuld?; Der UMGANG MIT DEM Holocaust; Germans and Jews SINCE THE Holocaust; STERN, Im Anfang war Auschwitz; ANTISEMITISMUS IN DER POLITISCHEN KULTUR NACH 1945. 
Die Beschreibung und die Definition des Verhaltens und der Befindlichkeit von Massen ist zwar eher Aufgabe des Soziologen oder des Psychologen als des Historikers. Doch ohne auf die Komplexität des vorliegenden Problems einzugehen, das heißt ohne den überwältigenden Einfluß des Holocaust auf die israelische Bevölkerung und vor allem auf die Holocaust-Überlebenden sowohl aus Osteuropa als auch aus anderen Regionen zu berücksichtigen, sind die späteren Entwicklungen nicht zu begreifen. Dem von den Erfahrungen des Krieges geprägten politisch-moralischen Ethos der israelischen Bevölkerung und der Einwanderer aus Osteuropa ist dabei besondere Aufmerksamkeit zu schenken. Jeder Impuls im Zusammenhang mit Deutschland rief bei Israelis umgehend eine - rationale oder irrationale - von Emotionen begleitete Reaktion hervor. Emotionen kristallisierten sich zu politischen Realitäten, wie schon so oft in der Geschichte. Doch trotz negativer Emotionen gingen die israelisch-jüdisch-deutschen Kontakte über eine Reihe von Themen weiter.

\section{Die deutsche Auseinandersetzung mit Israel}

Nach vier Jahren - nicht unumstrittener - halbpaternalistischer Besatzungsverwaltung der Westmächte war am 24. Mai 1949 das Grundgesetz für die Bundesrepublik Deutschland in Kraft gesetzt worden. Vier Monate später, am 21. September, erlangte der neue Staat beschränkte Souveränität, nachdem der erste Bundeskanzler, Konrad Adenauer, am Vortag seine Regierung und Politik vorgestellt hatte. Die Sowjetunion reagierte darauf am 7. Oktober 1949 mit der Gründung der Deutschen Demokratischen Republik. Beide neuen deutschen Staaten mußten die Folgen der gemeinsamen Vergangenheit fortan selbst tragen. Beide Staaten, vor allem aber die Bundesrepublik, hatten die Last der Eingliederung von Millionen von Flüchtlingen und Vertriebenen zu tragen und Tausenden von DPs eine Unterkunft bereitzustellen. Obwohl sich diese äußerst heterogene Flüchtlingsgemeinde zum Teil aus jüdischen Flüchtlingen zusammensetzte, schenkten die deutschen Behörden den Folgen der NS-Judenverfolgung nur geringe Aufmerksamkeit. Die deutschen Behörden auf Gemeinde-, Länder- und später auch auf Bundesebene konzentrierten sich vor allem auf die Probleme der "eigenen" Bevölkerung - auf Ernährung, Arbeit, Bereitstellung von Wohnraum und später auch auf den Wiederaufbau der nationalen Wirtschaft. Westdeutschland war dringend auf Devisen, Arbeitsplätze, Ausfuhr und auf die Wiederherstellung der eingebüßten Geltung in der Völkergemeinschaft angewiesen. Zuerst galt es, die Kreditwürdigkeit und vor allem das moralische Ansehen wiederherzustellen, um andere Staaten und Handelspartner vom guten Willen der neuen Republik zu überzeugen. So wurde die politische Führung der Bundesrepublik zwangsläufig wieder mit dem jüdischen Problem konfrontiert, nachdem es vier Jahre eher beiläufig wahrgenommen worden war.

Doch die Initiative in dieser Angelegenheit ging nicht von deutschen Politikern aus: Am 18. Mai 1949 wurde John McCloy zum Hochkommissar für Deutschland ernannt. In seiner Funktion als stellvertretender Kriegsminister der USA war McCloy während des Zweiten Weltkriegs wegen seiner Art, mit Minderheiten - 
Japanern und Juden - umzugehen, in Verruf geraten. Er war unter anderem auch an der Entscheidung beteiligt gewesen, die NS-Konzentrationslager nicht zu bombardieren. Möglicherweise fühlte er sich in seiner neuen Stellung deshalb verpflichtet, seine vormals negative Haltung gegenüber den Juden zu kompensieren. Anläßlich seiner ersten Begegnung mit jüdischen Vertretern in Deutschland am 31. Juli 1949 in Heidelberg, etwa drei Wochen nach seiner Ankunft, fand er folgende scharfen Worte:

„Das Verbrechen an dieser jüdischen Gemeinschaft führt uns zu den dunkelsten Kapiteln der Menschheitsgeschichte zurück. Welche Bedeutung diese Gemeinschaft in Zukunft erlangen wird, wie sie sich entwickelt und welchen Platz sie im neuen Deutschland einnimmt, wird die ganze Welt mit größter Aufmerksamkeit verfolgen, davon bin ich überzeugt. Dies wird meiner Meinung nach einer der Prüfsteine der Rückkehr Deutschlands zum Licht darstellen. ${ }{ }^{17}$

Hochkommissar McCloy verfolgte die jüdischen Angelegenheiten mit großem Interesse. Sein Berater für jüdische Fragen, Harry Greenstein, verstärkte diese Neigung und machte seinem Vorgesetzten, mit dem er ein einvernehmliches Verhältnis unterhielt, wiederholt auf das deutsche Schweigen hinsichtlich jüdischer Fragen aufmerksam. „Ich betonte die Tatsache, daß sich bis zum jetzigen Zeitpunkt kein einziger führender deutscher Politiker der Nachkriegszeit vom brutalen NS-Konzept minderwertiger Rassen distanzierte, und machte meinen Standpunkt deutlich, wonach eine Erklärung der gegenwärtigen Führung der Bundesrepublik Deutschland in diesem Sinne überfällig sei“, berichtet Greenstein in einem Schreiben vom 20. September 1949.18 McCloy beschloß zu handeln, nachdem er sich der Unterstützung des britischen und französischen Hochkommissars versichert hatte.

In seiner ersten Rede vor dem Bundestag äußerte sich der Kanzler zu jüdischen Angelegenheiten nur ganz knapp. Den Antisemitismus verurteilte er in zwei kurzen Sätzen:

„Lassen Sie mich [...] ein Wort zu hier und da anscheinend hervorgetretenen antisemitischen Bestrebungen sagen. Wir verurteilen diese Bestrebungen auf das Schärfste. Wir halten es für unwürdig und an und für sich unglaublich, daß nach all dem, was sich in nationalsozialistischer Zeit begeben hat, in Deutschland noch Leute sein sollten, die Juden deswegen verfolgen oder verachten, weil sie Juden sind. “19

Adenauers Rede enthielt weder eine explizite Verurteilung der deutschen Vergangenheit noch ein Ausdruck des Mitgefühls für die Opfer. Von Entschädigung der Opfer oder Bestrafung der Täter kein Wort. Auf jüdischer Seite reagierte man mit

17 Protokoll der Konferenz über „Die Zukunft der Juden in Deutschland“ vom Büro des Beraters für jüdische Angelegenheiten, Heidelberg, mit Randbemerkungen von John J. McCloy, Hoher Kommissar der amerikanischen Zone in Deutschland, S. 20, Truman Library, Papers of Harry N. Rosenfield, Box 16, Alphabetical File.

18 Harry Greenstein an Moses A. Leavitt vom 20.9. 1949, AJDC, AR-4564; vgl. dazu: Greenstein an McCloy vom 19. 9. 1949, AJDC, AR-4564, Nr. 377.

19 YIVO, AJC, FAD-1, Box 34, 20.9. 1949; VeRHANDLUNGEN DES DEUTSCHEN BUNDESTAGES, 1. WP 1949, Stenographische Berichte, Bd. 1, S. 27. 
Bedrückung und Irritation. ${ }^{20}$ Man hatte nicht erwartet, daß sich der neue Kanzler über die leid- und schmerzvolle Erfahrung der Juden so leicht hinwegsetzen würde. Die Sozialdemokraten erkannten den Affront und versuchten, den gravierenden Fehler wiedergutzumachen. Am folgenden Tag nahm der SPD-Parteivorsitzende Kurt Schumacher ausführlich zu Adenauers Ausführungen und zu den jüdischen Forderungen Stellung. ${ }^{21}$ Doch Adenauer ging hierauf nicht ein und hielt sich im Bundestag mit öffentlichen Äußerungen über Juden zurück. In einer Grußbotschaft zum jüdischen Neujahr (1949) nahm der Kanzler dann jedoch zusammen mit Bundespräsident Theodor Heuss öffentlich zu jüdischen Angelegenheiten Stellung. ${ }^{22} \mathrm{Ob}$ diesen Äußerungen Interventionen der Hohen Kommissare vorausgegangen waren, steht dahin. Aus dem Tagebuch von Adenauers engem Mitarbeiter Herbert Blankenhorn geht jedenfalls hervor, daß McCloy mit Vertretern der Bundesregierung das jüdische Problem wiederholt erörterte. ${ }^{23}$

Im Rahmen der im Kanzleramt stattfindenden langfristigen politischen $\mathrm{Pla}$ nung hatte die Frage der Wiedergutmachung der NS-Verbrechen seit der Regierungsbildung gebührende Aufmerksamkeit erhalten. Blankenhorn notierte folgendes:

„In diesen Gesprächen ist immer wieder der Gedanke vertreten worden, daß der neue deutsche Staat in der Welt Vertrauen, Ansehen und Glaubwürdigkeit nur wiedergewinnen werde, wenn die Bundesregierung und das Bundesparlament durch einen in freier Entscheidung getroffenen Willensakt sich von der Vergangenheit distanziere und durch eine eindrucksvolle materielle Wiedergutmachungsleistung dazu beitrage, das unglaubliche Ausmaß erlittener seelischer und materieller Not zu erleichtern und denen, die alles verloren hatten, beim Aufbau einer neuen Existenz zu helfen. "24

Blankenhorn hatte McCloys Heidelberger Erklärung fast vollständig übernommen. Die politische Elite in der Bundesrepublik Deutschland war von der Erkenntnis durchdrungen, daß die politischen Umstände eine Entspannung im Verhältnis zu den Juden unbedingt erforderten. Der Bundeskanzler brachte dies auch in einem Gespräch mit den Alliierten Hohen Kommissaren am 17. November 1949 zum Ausdruck. Hierin gab Adenauer die Versicherung ab, ,auf diesem Gebiete alles zu tun, was überhaupt möglich" sei. Der Bundeskanzler stellte in Aussicht, im Bundesinnenministerium ein „besonderes Referat für jüdische Fragen zu

20 „Denkschrift“, am 20.9. 1949 Chaim Yachil übergeben, Nachlaß Karl Marx (Privatbesitz), Kopien daraus im Besitz des Autors; LÜTH, Die Friedensbitte, S. 102 und 105.

21 Die SPD-Fraktion im Deutschen Bundestag, S. 11; vgl. die Rede Schumachers im Bundestag am 21. 9. 1949: VeRHANDLUNGEN DES DEUTSCHEN BundESTAGES, 1. WP 1949, Stenographische Berichte, Bd. 1, S. 31-42. Stellungnahme zur jüdischen Frage, S. 36; vgl. auch das Gespräch des Autors mit Norbert Wollheim am 3. 5. 1988 in New York; LüTH, Die Friedensbitte, S. 102-105.

22 Greenstein an McCloy vom 19. 9. 1949; Greenstein an Leavitt vom 20. 9. 1949, AJDC, AR 4564, No. 377; Eintrag vom 25. 9. 1949, BArch, N 1351, Bd. 1b; ALLGEMEINE WocHENZEITUNG der Juden in Deutschland vom 22. 9. 1949.

23 BArch, N 1351, Bd. 1 b.

24 BlANKENHORN, Verständnis, S. 138. 
errichten“, um damit „den in Deutschland lebenden Juden das Vertrauen zu geben, daß sie Schutz haben“. ${ }^{25}$

Wenige Tage vorher hatte der Bundeskanzler dem Chefredakteur der Allgemeinen Wochenzeitung der Juden in Deutschland (AWJD), Karl Marx, ein Interview gewährt, nachdem er zuvor das Gespräch mit ihm gemieden hatte. Hierin versprach Adenauer, den Rassismus und den Antisemitismus zu bekämpfen, dem Rechtsradikalismus energisch entgegenzutreten und jüdische Einrichtungen vor Übergriffen zu schützen. Ferner äußerte er die Bestrebung, den wirtschaftlichen Schaden wiedergutzumachen, den die Bürger jüdischen Glaubens im Dritten Reich erlitten hatten, und auf eine Verbesserung der Gesetze betreffend Restitution und Entschädigung hinzuwirken. Den Staat Israel bezeichnete er als Vertreter des Weltjudentums und offerierte als vorläufige symbolische Geste deutscher Entschädigungsbereitschaft gegenüber den Juden die Summe von zehn Millionen Mark. ${ }^{26}$

Marx war über Adenauers Äußerungen, besonders über sein Geldangebot, alles andere als erfreut. Ein Sprecher der israelischen Regierung lehnte es noch am selben Abend $a b$. An dieser Haltung konnten auch Adenauers nachträgliche Klarstellungen und Erläuterungen nichts ändern, wie etwa das Argument, die Besatzungsmächte seien gegen Zahlungen der Bundesrepublik Deutschland an andere Staaten. Israelischerseits wurde Adenauers Angebot zum Teil als Affront empfunden, als billiger Versuch, sich der Rückgabe geraubten Eigentums zu entziehen. Ihm wurde sarkastisch vorgehalten, sein Vorschlag laufe darauf hinaus, 1,66 Mark für jeden getöteten Juden zu zahlen. Für die ablehnende Haltung der Israelis gegenüber Adenauers Angebot gab es mancherlei Gründe. Mißtrauen war nur einer davon. Die Israelis waren zweifellos überrascht worden. Man hatte nicht mit dieser Geste gerechnet und wußte nicht, wie darauf zu reagieren war. Angesichts verbreiteter Haßgefühle gegenüber Deutschland rechnete niemand mit der Möglichkeit direkter Gespräche zwischen beiden Seiten. Zwar wurden einzelne Entschädigungsforderungen erhoben, doch ein detaillierter Forderungskatalog existierte zu diesem Zeitpunkt noch nicht, eine kollektive Entschädigung aus Deutschland stand noch kaum zur Debatte.

In einem Schreiben vom 10. Mai 1949 machte der israelische Gesandte in den Beneluxstaaten, Michael Amir, seinen Vorgesetzten vier Monate vor dem Amtsantritt der ersten Bundesregierung den Vorschlag, die Möglichkeit des Erhalts von Reparationen aus Deutschland zu prüfen. Amir argumentierte, die zukünftige deutsche Regierung würde die Wiederaufnahme in die Völkergemeinschaft anstreben und bereit sein, dafür einen Preis zu zahlen. Amirs Idee wurde von den Rechtsberatern des israelischen Außenministeriums kurzerhand verworfen. Es sei zu früh, sich mit einer Frage zu beschäftigen, die von der gegenwärtigen politischen Realität weit entfernt liege, hieß es. Auch detailliertere Darstellungen Amirs konnten den Argwohn der Juristen nicht beseitigen. ${ }^{27}$ Nach dem Treffen zwi-

25 Adenauer und die HoHen Kommissare, S. $25 \mathrm{f}$.

26 Der DeUTSCH-ISRAelische Dialog, S. 17-18.

27 Amir an Raday vom 10. 5. 1949; Rosenne an Amir vom 20. 6. 1949; Amir an Rosenne vom 11. 7. 1949, ISA, 2417/4. 
schen Bundeskanzler Adenauer und Nahum Goldmann, das im Dezember 1951 in London stattfand, lenkte Amir die Aufmerksamkeit des israelischen Außenministers Moshe Sharett erneut auf seinen Vorschlag. Sharett gab vor, nichts davon gewußt zu haben, und fügte hinzu, für diese Angelegenheit sei es überdies noch zu früh, niemand würde sie ernst nehmen. ${ }^{28}$

Die israelische Historikerin Yehudith Auerbach meint, Ministerpräsident Ben Gurion habe eine klare israelische Antwort auf Adenauers Angebot im Hinblick auf die zukünftigen Entwicklungen bewußt vermieden. Er sei bemüht gewesen, Israel nicht in die Kontroverse der Supermächte über Deutschland zu verwickeln, und er habe fest damit gerechnet, daß Deutschland früher oder später wieder in die Völkergemeinschaft aufgenommen werde: „Seine ständige Sorge um die Isolation Israels neben einer nüchternen Beurteilung von Deutschlands wieder zunehmender Bedeutung waren grundlegend für Israels Deutschlandpolitik [...] $\mathrm{Er}$ nahm das Thema Reparationen als Instrument zur Anbahnung bilateraler Verhandlungen wahr", schreibt Auerbach. ${ }^{29}$ Die vorhandenen Dokumente stützen diese These nicht. Sie deuten eher auf Moshe Sharett als israelischen Wegbereiter der Gespräche zwischen Westdeutschland und Israel hin. ${ }^{30}$ Amir trug seine Gedanken Sharett vor, und der Briefwechsel zwischen ihnen reflektiert die damalige israelische Haltung in dieser Frage: Die Zeit war noch nicht reif für Adenauers Idee. Einige Mitarbeiter deutscher Herkunft des JAFP, die sich häufig in Deutschland aufhielten, vertraten eine nüchternere Haltung und erkannten die große Bedeutung von Adenauers Angebot, doch noch bildeten sie die Minderheit.

\section{Reparationen, Restitution und Entschädigung}

Die Idee von Reparationen und der Restitution geraubten oder requirierten Eigentums durch den besiegten Feind ist so alt wie die Zivilisation selbst. So rief etwa der Ausbruch des Zweiten Weltkriegs Erinnerungen an die nach dem Ende des Ersten Weltkriegs abgeschlossenen Friedensverträge wach. Jüdische Flüchtlinge aus Deutschland, die alles zurückgelassen hatten, wogen sich in der Hoffnung, bald wieder in ihre Eigentumsrechte eingesetzt zu werden. Von der raschen Niederlage des Dritten Reichs überzeugt, schuf die JAFP das sogenannte Department for Recovery of Property of German Jews (DRPGJ) (Abteilung für die Wiedergewinnung des Eigentums deutscher Juden). Diesbezügliche Pläne nahmen bereits im September 1939 Gestalt an. ${ }^{31}$ In entsprechenden Briefwechseln ist von Entschädigungsansprüchen, Schadenersatzansprüchen, Hitlerschäden etc. die Rede, womit ausschließlich individuelle Forderungen gemeint waren. In manchen Briefen wurde die JAFP aufgefordert, die jüdischen Kriegsinteressen zu artikulieren und die Umsetzung der gesteckten Ziele vorzubereiten. Daher rührten das

${ }^{28}$ Amir an Sharett vom 28. 1. 1952; Sharett an Amir vom 28. 1. 1952, ISA, 2417/4.

29 AUERBACH, Ben Gurion and reparations, S. 275, 277-278, 283.

30 Sharett an Eban vom 14. 4. 1951, ISA, 5935/40; WEIrZ, Ben Gurions Weg, S. 255-279.

31 Erich Cohen an Georg Landauer vom 13. 9. 1939; Fritz Loewenstein an Siegfried Moses vom 17. 9. 1939, CZA, S 35/16. 
Auftauchen kollektiver Forderungen und die Verwendung von Ausdrücken wie "Jüdische Reparationsforderungen" und "Wiedergutmachung". Schalom AdlerRudel, ein zionistischer Aktivist deutscher Herkunft, versuchte verschiedentlich, führende zionistische Persönlichkeiten, darunter den Staatsmann und Biochemiker Chaim Weizmann, auf dieses Thema aufmerksam zu machen. ${ }^{32}$ Diese Briefe und Memoranden gelten als Wegbereiter des jüdischen Standpunkts in der Reparationsthematik.

Der deutsch-jüdischen Initiative schlossen sich in der Folge auch andere jüdische Emigrantengemeinden an, besonders die österreichische und die tschechoslowakische. Die erste Welle solcher Vorstöße ebbte 1941-42 ab, nahm jedoch 1943-44 verstärkt wieder zu. Auch jüdische Organisationen, darunter das American Jewish Committee (Committee for Peace Studies) und der Jüdische Weltkongreß setzten Untersuchungen über die Kriegsfolgen in Gang: 1943 gründete Bruno Weil, ein jüdischer Aktivist amerikanisch-deutscher Herkunft, die Axis Victims League, eine Organisation, die dann in den fünfziger Jahren zum Ärger der großen jüdischen Organisationen versuchte, auf Kosten einer einheitlichen jüdischen Haltung einen separaten Dialog mit Bonn zu führen. ${ }^{33}$

Öffentlich wurde das Thema Kriegsentschädigung für Juden zum ersten $\mathrm{Mal}$ von Nahum Goldmann im November 1941 anläßlich einer Tagung der Panamerikanischen Konferenz des Jüdischen Weltkongresses in Baltimore, Maryland, angesprochen. ${ }^{34}$ Am 5. Januar 1943 unterzeichneten sechzehn alliierte Regierungen und das French National Committee, die französische Exilregierung in London, die „Inter-Allied Declaration Against Acts of Dispossession Committed in Territories under Enemy Occupation or Control" (Interalliierte Erklärung gegen die Enteignungen in feindlich besetzten oder kontrollierten Gebieten). ${ }^{35}$ Die Exekutive der JAFP befaßte sich am 12. März und 31. Mai 1943 in London eingehend mit der Reparationsfrage und beschloß, einen Ausschuß mit der weiteren Bearbeitung dieser Angelegenheit zu beauftragen. Im Herbst desselben Jahres übertrug die JAFP diese Aufgabe dem neugebildeten Vorbereitungsausschuß für Nachkriegsplanung. ${ }^{36} \mathrm{Zu}$ den vom Ausschuß projektierten Einnahmequellen gehörten auch deutsche Reparationen. Den ersten systematischen Versuch, die Möglichkeiten deutscher Zahlungen auszuloten, unternahm das im März 1941 vom Jüdischen Weltkongreß in New York gegründete und von den Brüdern Jacob und Nechemiah Robinson geleitete Institute of Jewish Affairs. Die beiden Persönlichkeiten genossen internationalen Ruf im Hinblick auf die schwierige Materie Reparationen, Entschädigung und Eigentumsrückerstattung. Schließlich verfaßte der deutsch-jüdische Autor Siegfried Mozes den 1944 veröffentlichten „Aufsatz über die Wiedergutmachung der Juden“. Diese Schrift sollte die jüdische Haltung in

32 Adler-Rudel, Aus der Vorzeit, S. 200-204; vgl. auch The LetTers AND PAPERS OF Chaim WeizmanN, Jerusalem, Series A, Bd. 19, 210, Dok. Nr. 188.

33 Bruno Weil an Dr. F. Goldschmidt, o.D., [verm. 24. 10. 1951], LBI, Council for the Protection of Rights and Interests of Jews from Germany (CPRIJG), Folder 2, Council, M/m, Haag Conference, bis 31. 5. 1952.

34 GoldmanN, Generation, S. 171-178.

35 Text der Erklärung: CZA, A 140/497.

36 AdLER-RudeL, Aus der Vorzeit, S. 210-211. 
dieser Frage nachhaltig beeinflussen, doch sie war nicht die einzige: Der Direktor der DRPGJ, Georg Landauer, hatte bereits im November 1943 ein internes Memorandum mit dem Titel „Die jüdischen Nachkriegsforderungen“ vorgelegt, das die Haltung der JAFP beeinflußte. ${ }^{37}$

Ein Teil des Eigentums, das jüdische Flüchtlinge im Dritten Reich zurücklassen mußten, konnte vor Ausbruch des Krieges durch den Transfer nach Palästina gerettet werden. Dieselben Personen, die sich vor dem Krieg mit diesem Eigentumstransfer, der sogenannten Haavarah (hebr. Transfer, Überführung) ${ }^{38}$, beschäftigt hatten, nahmen sich auch nach Kriegsende der jüdischen Forderungen gegenüber Deutschland an. Die mit dem Transfer von Eigentum nach Palästina beauftragt gewesene Firma „Nir" entwarf bereits während des Krieges Pläne für die Rückgewinnung jüdischen Eigentums und die Zahlung von Reparationen nach Kriegsende. Ein faszinierendes Element dieser Planung bildete der Briefwechsel zwischen dem britischen Geschäftsmann Israel M. Sieff und dem amerikanischen Fachmann für Emigrationsfragen, Eli Ginsburg. In einem nicht genau datierten Brief aus dem Jahre 1943 erörtert Ginsburg die Möglichkeit der Verwendung deutscher Reparationen zur Umsiedlung palästinensischer Araber. ${ }^{39}$

Das Planungskomitee beendete seine Arbeit im Jahre 1944. Sein Subkomitee für Reparationen unterbreitete den Vorschlag, bei der deutschen Regierung einen formellen Antrag auf kollektive Entschädigung im Namen des jüdischen Volkes einzureichen und individuelle Forderungen zu erheben. Dabei ging man davon aus, daß die Reparationen vor allem in Form von Güterzahlungen erfolgen würden, wovon zwangsläufig nur die jüdische Gemeinschaft in Palästina profitieren könnte. Zudem sollte Deutschland, gemäß diesem Vorschlag, auch die Auswanderung von Juden nach Palästina finanzieren. Die Alliierten seien ferner von der Existenz einer jüdischen Nation mit Anrecht auf kollektive und individuelle Entschädigung zu überzeugen. ${ }^{40}$ Die überwiegende Mehrheit dieser Pläne lief also auf Vorschläge hinaus, Deutschland finanziell für den Aufbau der nationalen Heimstätte des jüdischen Volkes in Palästina heranzuziehen.

Im November 1944 berief der Jüdische Weltkongreß in Atlantic City, New Jersey, eine Kriegsnotkonferenz über das Schicksal des europäischen Judentums ein, auf der verschiedene Resolutionen über Entschädigung und Restitution verabschiedet wurden. Die Umsetzung dieser Resolutionen nach dem Krieg muß

37 LANDAUER, Die jüdischen Nachkriegsforderungen.

38 JAFP-Projekt der Jahre 1937-38 zur Rettung jüdischer Eigentumswerte im Dritten Reich. Im Rahmen dieses Projekts lieferte Nazideutschland Güter nach Palästina, deren Erlös an die Neueinwanderer aus Deutschland verteilt wurde. Im Gegenzug verpflichteten sich die Neueinwanderer auf ihr Eigentum in Deutschland zu verzichten.

39 Der Brief lag einem Memorandum des America-Palestine Institute vom 11.12. 1943 bei: F. D. Roosevelt Library, New York, Louis Bean Papers. Für den Hinweis auf dieses Dokument bin ich Prof. Dr. Helmuth Mejcher von der Universität Hamburg zu Dank verpflichtet.

40 Landauer an Mozes, Rosenbluth und Kreutzberger vom 25. 9. 1944, LBI, Nachlaß Landauer, B26/1, Nr. 16; Das Subcommittee (for reparations) of the Planning Committee an das Planning Committee vom 29. 9. 1944, Ausarbeitung von Walter Turnowsky, „Pläne für Einwanderung in großem Maßstab“ o.D., Dr. D. Azian, Sekretär der Planungsgruppe, an Mitglieder der Planungsgruppe vom 5., 12. und 16. 10. 1944, BGA, Correspondence Files. 
jedoch in den Zusammenhang der neu auftauchenden jüdischen Probleme, wie etwa dem Problem der entwurzelten jüdischen Gemeinschaften (Displaced Persons), des britisch-jüdisch-arabischen Verhältnisses in Palästina sowie der Spannungen zwischen den Alliierten gestellt werden. Später stellte sich heraus, daß die Alliierten den jüdischen Forderungen kaum Rechnung trugen. Obschon die Juden ein Hauptopfer des Krieges waren, besaßen sie keine eigene, unabhängige politische Vertretung. Die Konferenz von Jalta erklärte nur Staaten, die am Krieg beteiligt gewesen waren oder unter deutscher Besetzung gestanden hatten, als berechtigt zum Erhalt von Reparationen. Trotz Memoranden und Appellen jüdischer Organisationen, darunter der JAFP, und verschiedener jüdischer Persönlichkeiten an die Siegermächte und an internationale Organisationen, befaßte man sich weder in Jalta noch auf der Potsdamer Konferenz mit den jüdischen Wiedergutmachungsansprüchen. Bernhard Joseph, ein Vertreter der JAFP, die sich berufen fühlte, die Sache des jüdischen Volkes zu vertreten, verfaßte eine längere eidesstattliche Erklärung zu den jüdischen Forderungen, die auf internationalem Recht basierte. Gestützt auf diese Erklärung überreichte die JAFP den Alliierten am 20. September 1945 ein offizielles Protestschreiben, das die Unterschrift von Chaim Weizmann trug. Appelle in ähnlichem Sinne hatte diese Organisation zuvor schon im Oktober 1944 und im Mai 1945 veröffentlicht. ${ }^{41}$ Das Resultat war jedoch mager und ging kaum über Sympathiebekundungen hinaus. Die Juden wurden weiterhin als Individuen - entweder als Bürger bestimmter Staaten oder als Staatenlose - ohne Anspruch auf kollektive Entschädigung betrachtet. ${ }^{42}$

In der Frage der deutschen Reparationen herrschte Uneinigkeit zwischen den Großmächten. Die Westmächte kalkulierten langfristig und betrachteten das zukünftige Verhältnis zu Deutschland aus der Warte der von der Sowjetunion ausgehenden kommunistischen Bedrohung. Der besiegte Feind von gestern sollte zum Verbündeten von morgen werden, daher das Interesse an seiner möglichst raschen wirtschaftlichen Erholung. Zu hohe Reparationen wären dabei kontraproduktiv gewesen. Die Erinnerungen an die Folgen des Versailler Vertrages waren noch lebendig, und deren Wiederholung wollten die Alliierten um jeden Preis verhindern, auch um ein erneutes Gedeihen des Extremismus zu verhindern. ${ }^{43}$

Während Großbritannien und die USA auf ihre Reparationsforderungen praktisch ganz verzichteten und sogar Frankreich einem solchen aus eigener Sicht unbequemen Modus vivendi zustimmte, zeigte sich die Sowjetunion weit weniger kompromißbereit. Das Land war im Krieg schwer in Mitleidenschaft gezogen worden und forderte, Deutschland im allgemeinen und die westlichen Besatzungszonen im besonderen bis zum letzten Pfennig auszupressen. Doch die Westmächte kamen überein, das Reparationskapitel zu schließen und die Sowjetunion

41 Introduction to the Letter of 20 September 1945, Dr. Chaim Weizmann on behalf of the Jewish Agency for Palestine to the Governments of the United Kingdom, the United States, the USSR and France. In: WeIZMANN. Letters of Weizmann, Bd. 9 und Vol. 12, 11.

42 Robinson an Goldmann vom 17. 7. 1945, AJA, WJC, U-245; ZwEIG, German Reparations, S. 2-3.

43 Adenauer und die deutsche Frage, S. 15-18. 
daran zu hindern, es wieder aufzurollen. ${ }^{44}$ Indirekte Opfer dieses Gegensatzes waren die Juden. Die Westmächte behandelten die Reparationenfrage gleichsam als Büchse der Pandora und waren nicht gewillt, der UdSSR ihr Öffnen zu erleichtern. Daß sich dieses Problem im Rahmen des späteren Friedensvertrages regeln ließe, wurde zumindest in jenen frühen Jahren noch für möglich gehalten. Doch eine unmittelbare Lösung für die von den Juden erhobenen Ansprüche war damit kaum gegeben. Versuche von jüdischer Seite, Deutschland zur Zahlung von Entschädigung zu bewegen, stießen bei den Alliierten auf wenig Gegenliebe, sowohl aus globalstrategischen Erwägungen als auch aus Furcht vor der sowjetischen $\mathrm{Re}$ aktion. In den Jahren 1944 und 1945 kam die Frage der Entschädigung für Juden im amerikanischen Außenministerium zur Sprache, doch diese Debatten verliefen im Sand. Darauf wollen wir später noch zurückkommen.

Solange die jüdische Gemeinschaft in Palästina gegen das britische Mandat ankämpfte und sich dabei auch in einer Konfrontation mit der arabischen Welt befand, hätte eine Zustimmung für deutsche Reparationen an Juden direkt gegen britische Interessen verstoßen. Solche Reparationen hätten Öl ins Feuer dieses Konflikts gegossen, was man in London unbedingt verhindern wollte. $\mathrm{Zu}$ diesem Zeitpunkt bestand also keine Aussicht auf kollektive Entschädigung für die Juden. Die Reparationsfrage wurde nach 1945 ad acta gelegt und erst 1950 wieder aufgerollt. Jüdische Kritiker sprachen von fünf verlorenen Jahren.

In jener Zeitspanne wurden die katastrophalen Ausmaße des Holocaust allmählich bekannt. Die erdrückende Last der Wahrheit über diese Tragödie erzeugte gewaltigen moralischen Druck, was jedoch vorerst nicht zu konkreten Maßnahmen führte. Nur vereinzelt wurde die Reparationsfrage von Gruppierungen und bestimmten Persönlichkeiten weiterverfolgt. So forderte etwa das American Jewish Committee (AJC) indirekt über die amerikanischen Behörden deutsche Hilfe für die Emigration jüdischer NS-Opfer. Ähnliche Forderungen hinsichtlich deutscher Kostenbeteiligung wurden auch vom sich direkt um die jüdischen Flüchtlinge kümmernden American Joint Distribution Comittee (AJDC) und von der für die Emigrationsvorkehrungen von DPs zuständigen JAFP erhoben. Die zwischen November und Dezember 1945 stattfindende Pariser Reparationenkonferenz honorierte diese Anstrengungen mit der Zuweisung von 25 Millionen Dollar aus deutschen Guthaben im Ausland sowie einem Teil des in Deutschland beschlagnahmten oder sichergestellten Goldes für "nicht repatriierbare" Flüchtlinge. AJDC und JAFP erhielten $90 \%$ dieser Summe für die Finanzierung der Emigration jüdischer Flüchtlinge. Auch die Internationale Flüchtlingsorganisation (IRO) übernahm gewisse Aufgaben für die jüdischen Flüchtlinge. ${ }^{45}$ Das am 14. Juni 1946 unterzeichnete Konferenzabkommen sprach den Juden erstmals

44 Vertrauliches Memorandum von Mr. Tremble vom 23. 6. 1951, PRO, FO 371/93516.

45 Entwurf der Ausarbeitung „Der Beitrag des AJC zur ökonomischem Wiedergutmachung von jüdischen NS-Verfolgten" vom 1.9. 1965, YIVO, AJC, Institute for Human Affairs, RG-1, EXD-20, JSX63; Ausarbeitung von Simor Segal „Reparationen“ vom 2. 4. 1946, YIVO, AJC, RG-347, GEN-10, Box 276; Ginsberg, Eli: Reparations for Non-Repatriables. In: Department of State Bulletin vom 14. 7. 1946, Final Act of the Paris Conference on Reparations, London, H.M. Stationery Office, 1946, S. 213-215, CZA, A 140/555. 
separate Reparationen zu, wenn auch nicht nach den Konzepten und Vorstellungen jüdischer Kreise. Zudem wurde deutsches Grundeigentum in Palästina zum potentiellen Reparationsobjekt erklärt, worauf später noch einzugehen sein wird.

Individuelle Forderungen fußten auf einer sicheren Rechtsbasis und wurden in den westlichen Besatzungszonen - in unterschiedlichem Maße - auch durchgesetzt. Solche Ansprüche waren aber oft mit langwierigen gerichtlichen Verfahren verbunden, was einen JAFP-Funktionär 1946 dazu bewog, eine globale Lösung vorzuschlagen. Vertreter der US-Regierung waren dagegen, da sie befürchteten, eine Globalisierung könnte die Sozialisierung individueller Rechte hervorrufen. ${ }^{46}$ Trotzdem sollte die Idee der Globalisierung noch mehrere Male und in verschiedenen Formen auftauchen.

Auch prominente Persönlichkeiten in Israel nahmen die Reparationsproblematik wieder.auf. $\mathrm{Zu}$ ihnen gehörte etwa der nationalreligiöse israelische Politiker Zerach Warhaftig. Der jiddische Dichter H. Lewick rief 1949 indes die Holocaust-Überlebenden dazu auf, ihre Forderungen gegenüber Deutschland fallenzulassen und dieses Land zu boykottieren. ${ }^{47}$ Am 2. März 1949 beschäftigte sich die israelische Regierung kurz mit dem Thema. ${ }^{48}$ Die "fünf verlorenen Jahre" verstrichen also nicht ereignislos, wenn auch die jüdischen Forderungen in dieser Zeitspanne eine vergleichsweise untergeordnete Rolle spielten. Erst die Gründung der Bundesrepublik Deutschland erfüllte die Reparationsidee wieder mit Leben.

Es gab drei verschiedene Arten von jüdischen Forderungen: Restitution, Entschädigung (auf kollektiver und individueller Basis) sowie die sogenannte Dritte Masse. Die Restitution betraf Eigentum, das im Rahmen der Arisierungspolitik vom NS-Regime beschlagnahmt oder dem rechtmäßigen Besitzer zu äußerst unvorteilhaften Bedingungen zwangsweise abgekauft worden war. Obwohl diese Eigentümer größtenteils im Holocaust umgekommen waren, konnte ein Teil des Besitzes identifiziert werden. Überlebende Eigentümer oder ihre Erben versuchten, ihr ursprüngliches Besitzrecht wieder herzustellen. Zur Regelung dieses Bereiches erließen die Militärregierungen Restitutionsgesetze, deren Ausführung den Länderregierungen auferlegt wurde: Am 10. November 1947 traten in der amerikanischen Zone das Militärgesetz Nr. 59 und in der französischen Zone das Gesetz Nr. 120 in Kraft. Am 12. Mai 1949 folgte das Gesetz Nr. 59 in der britischen Zone, und zwei Wochen später, am 26. Mai, wurde in den Westzonen Berlins das so genannte Kommandanturgesetz erlassen. ${ }^{49}$ Verschiedene, die Interessen deutscher Juden vertretende Organisation gründeten zusammen das United Restitution Office (URO) zur Unterstützung individueller Forderungen und

46 Yachil an Jacob [Robinson?] vom 7. 1. 1952, ISA, 43/10.

47 Interview mit Zerach Warhaftig vom 14. 7. 1976, BGA, Oral History Division; Dawar (Tel Aviv) vom 1.1. 1952.

48 SEGEV, Die siebte Million, S. 289; SAGI, Wiedergutmachung für Israel; PROSS, Wiedergutmachung; GOSCHLER, Wiedergutmachung, Westdeutschland und die Verfolgten; BALABKINS, West German Reparations; BLESSIN, Wiedergutmachung; WIEDERGUTMACHUNG IN DER Bundesrepublik Deutschland; PeAse, After the Holocaust; Zweig, German Reparations.

49 Shinnar an Sharett vom 9. 1. 1952, ISA, 2417/4. 
zur Informierung der Anspruchsteller über die bestehende Restitutionsgesetzgebung..$^{50}$

Das Eigentum der im Holocaust Ermordeten war in den Händen derer geblieben, die es sich im Laufe der Arisierungskampagne unrechtmäßig angeeignet hatten, indem sie von den antijüdischen Maßnahmen der NS-Behörden profitierten. Die neuen Eigentümer schoben die Verantwortung für die Enteignung dem Dritten Reich und der NSDAP zu und betrachteten sich als rechtmäßige Eigentümer, die ihr Eigentum in gutem Glauben erworben hatten. In ihren Augen waren die Restitutionsforderungen deshalb ungerechtfertigt. Die alliierte Gesetzgebung in diesem Bereich variierte zwar in Qualität und Quantität, orientierte sich jedoch im allgemeinen an den Interessen der Enteigneten. Etwa ein Drittel der ehemaligen Eigentümer hatte sich inzwischen in Israel niedergelassen. ${ }^{51}$ Identifiziertes erbenloses Eigentum, gemeinschaftliches Eigentum jüdischer Institutionen, Stiftungen, Treuhandgesellschaften usw. waren von diesen Gesetzen nicht betroffen und verblieben somit in den Händen der unrechtmäßigen Besitzer, ganz nach dem in Israel und in jüdischen Kreisen damals so oft zitierten Ausspruch des (biblischen) Propheten Elias: „Du hast gemordet, dazu auch fremdes Gut geraubt“ (Könige I, 21,19). Zur Behebung dieses Mißstands wurden spezielle Organisationen ins Leben gerufen. Die erste war die Jewish Restitution Successor Organization (JRSO), ein Produkt langwieriger Verhandlungen zwischen dem AJC, dem Jüdischen Weltkongreß, der JAFP, dem AJDC, dem amerikanischen Außenministerium und der amerikanischen Militärregierung. Die JRSO wurde ermächtigt, nicht beanspruchtes und erbenloses jüdisches Eigentum sicherzustellen und zu verwalten. Dahinter verbarg sich die Idee, „Eigentum im Wert von Millionen Dollar zugunsten jüdischer Interessen sicherzustellen, das andernfalls in deutsche Hände gelangt wäre und nun endlich eine positive Verwendung finden soll...", wie es der JAFP-Funktionär Maurice Boukstein formulierte. ${ }^{52}$ Das sichergestellte Vermögen wurde schließlich zwischen dem AJDC und der JAFP aufgeteilt und diente dem Zweck der Rehabilitation von DPs sowie ihrer Integration in Israel. Dabei kam es zu Unstimmigkeiten zwischen den Organisationen für deutsche Juden, der JRSO, und den neuen jüdischen Gemeinden in Deutschland. ${ }^{33}$ Ähnliche Organisationen in der britischen und französischen Besatzungszone, die Jewish Trust Corporation und die JRSO hofften, gemeinsam Erlöse im Gesamtbetrag von rund drei Milliarden Mark zu erzielen. ${ }^{54}$

Jüdische Emigranten aus Deutschland und andere Opfer des Nationalsozialismus stellten diverse Ansprüche auf Entschädigung - für Freiheitsentzug, Körper-

50 Memorandum über die Tätigkeit des United Restitution Office (URO) vom 7. 2. 1952, ISA, 2543/12.

51 Landauer, Restitution of Jewish Property, S. 8.

52 BGA, Protocols File, Maurice Boukstein, JAFP Executive Committee, American Section, January-June 1949.

53 JeLINEK, Leo Baeck, S. 236-241; Jelinek, Die Politik, 369-387.

54 KaPRaliK, Reclaiming the Nazi Loot; Memorandum des JRSO, „Möglicher Konflikt zwischen den Wiedergutmachungsansprüchen der israelischen Regierung gegenüber Deutschland und jüdischen Forderungen unter den existierenden deutschen Gesetzen zu Entschädigung und Rückerstattung vom Juli 1951, ISA, 2543/12. 
verletzung, Erniedrigung, wirtschaftlichen Schaden, für zunichte gemachte Studien- und Berufsperspektiven etc. DPs in der amerikanischen Zone erhielten Entschädigung für die Gefangenschaft in Ghettos, Gefängnissen und Konzentrationslagern, gebunden an gewisse zeitliche Grenzen und gestützt auf unter alliierter Besatzungsherrschaft verabschiedete Ländergesetze, in Kraft getreten am 10. August 1949 in der amerikanischen Zone, Ende Mai 1950 in der ehemaligen französischen Zone und in den Berliner Westsektoren erst am 10. Januar 1957. Die Entschädigungsgesetze der britischen Zone blieben dagegen vorerst unzureichend, da die britische Regierung die Regelung der Entschädigung der Bundesrepublik überließ.55

Die URO beschäftigte sich anfänglich sowohl mit Restitution als auch mit Entschädigung. Später kümmerte sich eine spezielle Institution namens Miltam (ein hebräisches Achrostichon für ,israelisches Büro für Entschädigungsforderungen von Deutschland“) um die ehemaligen DPs. Nach deren Einrichtung wurden sämtliche rechtlichen Schritte und Entschädigungsklagen aller Art der URO überlassen. Die Kriegsentschädigungen entwickelten sich zu einem lukrativen Geschäft für Scharen von Rechtsanwälten und anderen Mittelspersonen, von denen nicht alle aufrichtig und ethisch handelten. Während am Anfang fast ausschließlich Organisationen der jüdischen Diaspora die jüdischen Interessen hinsichtlich Kriegsentschädigung und Eigentumsrückführung vertraten, nahm das diesbezügliche Interesse der israelischen Behörden erst in den frühen fünfziger Jahren allmählich zu.

Die „Dritte Masse“ betraf eine Vielfalt von kollektiven Forderungen bezüglich der Hinterlassenschaft des Dritten Reiches. Viele Juden in Deutschland und in den besetzten Ländern waren von den Nationalsozialisten auf verschiedenen Wegen ihres Eigentums beraubt worden. Zu erwähnen sind die Sondersteuer in Höhe von einer Milliarde Mark, die nach den Pogromen der „Kristallnacht" erhoben wurde, die Emigranten aufgebürdete "Reichsfluchtsteuer", die sich aus dem Transfer von Eigentum der Emigranten ergebenden Verluste und der in den besetzten europäischen Ländern beschlagnahmte Besitz der im Holocaust Umgekommenen. Dem wären noch die Gewinne aus Zwangsarbeit, Verarbeitung der Leichen von Holocaustopfern und vieles mehr hinzuzufügen. Die "dritte Masse“ sollte später die Rechtfertigungsbasis für jüdische Reparationsansprüche gegenüber Deutschland bilden. Die Ansprüche auf globale Entschädigung lösten jene Entwicklungen aus, die schließlich zu den sogenannten Schilumim, den Verhandlungen über Entschädigungszahlungen, führten. ${ }^{56}$

Eng mit der JRSO verbunden war die Jewish Cultural Reconstruction Company, die versuchte, kulturell, künstlerisch und religiös bedeutsame jüdische Kunstobjekte aus dem jüdischen Raubgut der Nationalsozialisten, darunter Bücher, dokumentarische Texte, Thorarollen, rituelle Gewänder und Gegenstände sowie Gemälde und Möbel, sicherzustellen bzw. zurückzuerhalten. Die oberste

55 Shinnar an Sharett vom 9. 1. 1952, ISA, 2417/4; GOSCHLER, Wiedergutmachung, Westdeutschland und die Verfolgten, S. 185-189.

56 Für eine Übersicht zur „Dritten Masse“: Küster an Adenauer, betr. Richtlinien für die Wiedergutmachungspolitik vom 12.7. 1952 (in Abschrift), ISA 2543/12. 
Leitung dieser Institution und die öffentliche Aufsicht oblag größeren jüdischen Organisationen mit namhafter deutsch-jüdischer Vertretung. Die Philosophin Hannah Arendt bekleidete den Direktorenposten. Es war die Aufgabe dieser Institution, die ursprünglichen Eigentümer der Kunstobjekte zu eruieren und ihnen ihr Eigentum zurückzugeben oder, falls dies nicht gelang, es unter den religiösen und kulturellen jüdischen Institutionen zu verteilen. ${ }^{57}$

Die Sicherstellung verlorenen Eigentums und die Beschaffung von Geldmitteln für die jüdische Besiedlung Palästinas war zu jener Zeit eines der Hauptziele für die Anknüpfung deutsch-jüdischer Beziehungen. Dabei wurde auf zwei Ebenen vorgegangen: Privatpersonen versuchten, ihre ursprünglichen Eigentumsrechte wiederherzustellen, während im öffentlichen Bereich Anstrengungen unternommen wurden, wenigstens einen Teil des geraubten Gemeindebesitztums sicherzustellen. Unter der alliierten Besatzung verliefen die Kontakte zwischen beiden Seiten meistens über die Militärbehörden. In diesem frühen Stadium kann deshalb kaum von direkten deutsch-jüdischen Beziehungen im Bereich der Finanzhilfe und der Eigentumsrückführung gesprochen werden.

\section{Die Akteure}

Mehrere Gruppen in Israel machten Ansprüche im Hinblick auf Reparationen, Entschädigung und Eigentumsrückerstattung geltend. Zu ihnen zählten besonders Neueinwanderer aus Deutschland, Holocaust-Überlebende, bestimmte Gruppen ehemaliger Beschäftigter des öffentlichen Dienstes in Deutschland, die ein Recht auf Rente beanspruchten, sowie die sich um Reparationen kümmernden Regierungsämter und JAFP-Stellen. Gleichzeitig vertraten Organisationen und Institutionen außerhalb Israels die Interessen der jüdischen Bevölkerung in den jeweiligen Ländern. Hinzuzufügen sind schließlich Instanzen, wie etwa die JRSO und die Jewish Cultural Reconstruction Company, die sich um die Rückführung spezifischen Eigentums kümmerten. Das Schicksal der während des Krieges beschlagnahmten und auch lange Jahre nach Kriegsende noch in deutscher Hand befindlichen umfangreichen jüdischen Eigentumswerte war ein häufiges $\mathrm{Ge}$ sprächsthema jüdischer und israelischer Funktionäre. Man war sich einig, daß dieses Eigentum, falls sichergestellt, der Wiedereingliederung und Repatriierung der Holocaust-Überlebenden zugeführt werden müsse. Auf der Tagesordnung stand die Idee einer kollektiven Abgeltung für das jüdische Volk.

Bis zum Herbst 1951 wurden die diesbezüglichen Verhandlungen gleichzeitig auf drei bis vier verschiedenen Ebenen geführt. Der über einflußreiche Vertretungen in den USA, in Israel und in Großbritannien verfügenden Jüdische Weltkongreß trat hier besonders in den Vordergrund. Die Hauptrollen bei ersten Vorgesprächen mit den britischen und deutschen Behörden spielten dabei der WJC-Präsident Nahum Goldmann, der stellvertretende Präsident der britischen

57 Jüdisches Kultureigentum, AJDC, File 4266; Jerome Michael an I. L. Kenen vom 31. 1. 1947, YIVO, AJC, RG 347, GEN-10, Box 291. 
Vertretung des WJC, Noah Barou, und deren Sekretär, Alex Easterman. Eine wichtige Funktion kam sodann der über Vertretungen in sämtlichen größeren jüdischen Zentren der Welt verfügenden JAFP zu. Goldmann amtierte als einer ihrer zwei Vorsitzenden und war somit das Verbindungsglied in persona zum Jüdischen Weltkongreß. Die JAFP unterhielt zudem ein Büro in München, das von Georg Landauer, dem ehemaligen Direktor des Central Bureau for the Settlement of German Jews und des DRPGJ, sowie von einem Funktionär namens Max Kreutzberger geleitet wurde, der die Tagesgeschäfte erledigte. Eine weitere aktive Organisation war die von New York aus operierende AJDC, eine apolitische (nicht-zionistische) Repräsentantin des amerikanischen Judentums. Als philanthropische Organisation mit Wohltätigkeits- und Wiedereingliederungsaufgaben war sie zwangsläufig mit Angelegenheiten konfrontiert, die Deutschland und die Überlebenden der NS-Verfolgung betrafen. Zahlreiche Initiativen gingen auch von den politisch bedeutsamsten Vertretern des amerikanischen Judentums, dem nicht-zionistischen AJC, dem American Jewish Congress, der Bnei Brit sowie dem Jewish Labour Committee aus. Unter diesen war das AJC am aktivsten. Sein Vertreter in Paris besaß gute Kontakte in Washington und bei den Militärbehörden in Frankfurt am Main. Jacob Blaustein, der Vorsitzende dieser Organisation, und deren Berater für auswärtige Angelegenheiten, Seymour J. Rubin, trugen die Hauptlast der Arbeit.

Auf amtlicher israelischer Ebene sorgte in erster Linie das Außenministerium unter Moshe Sharett für die Duchsetzung jüdischer Ansprüche sowie - im Ausland - die israelischen diplomatischen Vertretungen. Am 1. Januar 1950 wurde in der Nachrichtenabteilung des Außenministeriums ein deutsches Referat unter Viktor Fischl (später Avigdor Dagan) eingerichtet und 1951 in die Westeuropaabteilung unter Felix Eliezer Shinnar (ehemals Schneebalg) eingegliedert. Das israelische Konsulat in München und der bei den Besatzungsbehörden in München akkreditierte Konsul Eliahu Kurt Livneh waren die führenden diplomatischen Außenposten Israels für deutsche Angelegenheiten. Von dieser Vertretung wurden inoffizielle Gespräche geführt, Informationen gesammelt und Berichte nach Israel gesandt. ${ }^{58}$

Eine weitere aktive Regierungsinstitution war das für die wirtschaftlichen und finanziellen Aspekte zuständige Finanzministerium unter Finanzminister Elieser Kaplan. Der Anteil von Ministerpräsident David Ben Gurion und seines Amtes beschränkte sich dagegen auf die bloße Beschlußfassung auf höchster Stufe.

Unter den vier führenden mit Entschädigungs-, Restitutions- und Wiedergutmachungsfragen befaßten jüdischen Persönlichkeiten gab Goldmann zwar den Ton an, arbeitete aber dennoch eng mit Sharett zusammen. Blaustein war wegen seiner guten Beziehungen in Washington die Schlüsselfigur bei Verhandlungen mit der amerikanischen Regierung. Dagegen mutet Ben Gurions Rolle vergleichsweise zweitrangig an. Die für Deutschland zuständigen Abteilungen der JAFP waren mit deutschen Emigranten besetzt. In manchen Fällen handelte es sich dabei um Veteranen der Haavarah. Sie unterhielten enge Beziehungen zu verschiede-

58 JELINEK, Like an Oasis, S. 81-98. 
nen deutsch-jüdischen Organisationen, wie etwa der Israeli Association of Immigrants from Central Europe (AICE) und dem von London aus operierenden Council for Protection of Rights and Interests of Jews from Germany (CPRIJG).

Manche leitenden Mitarbeiter im israelischen Außen- und Finanzministerium waren ebenfalls deutscher Herkunft. Zu nennen wären der Leiter der Westeuropaabteilung im Außenministerium, Gershon Avner, und der Direktor der israelischen Zollbehörde, Kurt Mendelssohn. Eine wichtige Rolle im Außenministerium spielten zudem Mitarbeiter österreichischer oder tschechoslowakischer Herkunft mit teilweise deutschem Hintergrund, wie zum Beispiel Chaim Yachil (ehem. Heinrich Hoffmann), Eliahu Kurt Livneh (ehem. Liebstein) und Avigdor Dagan. Ein zu beobachtender Antagonismus zwischen der JAFP und dem israelischen Außenministerium läßt sich zumindest teilweise durch die Mentalitätsunterschiede zwischen den genannten Personen und ihren Kollegen polnischer- bzw. russischer Herkunft und durch das unterschiedliche soziale Milieu dieser beiden Gruppen erklären. Doch die fast sprichwörtliche Animosität zwischen den beiden Institutionen lag zweifellos auch in der gegenseitigen Konkurrenz um Macht, Einfluß und Geltung begründet, die eine gegenseitige Distanz in deutschen Angelegenheiten hervorrief. Deutsche Instanzen waren sich dieser subtilen Differenzen kaum bewußt. Dies umso weniger, als die israelischen Regierungsvertreter strikte Anweisung hatten, den Kontakt mit deutschen Stellen jeder Art zu meiden, während JAFP-Vertreter keinen solchen Auflagen unterworfen waren. Für die deutsche Seite repräsentierte die JAFP somit faktisch den Staat Israel, und die Vertreter dieser Organisation waren nicht bemüht, ihren eigentlichen Status klarzustellen. Der Machtkampf belastete die tägliche Arbeit dieser beiden israelischen Instanzen und erforderte wiederholt das Eingreifen höchster Stellen.

Spannungen und Machtkämpfe charakterisierten auch das Verhältnis zwischen der JAFP und dem Jüdischen Weltkongreß, vor allem Deutschland betreffend. Die JAFP-Agenten strebten - bewußt oder unbewußt - das Monopol in deutschen Angelegenheiten an und setzten sich gegen jede "Einmischung“ anderer Organisationen zur Wehr. Das AJC besaß zudem ein problematisches Verhältnis $\mathrm{zu}$ anderen Organisationen, besonders zu den Vertretungen des Staates Israel. Die nicht-zionistische - damals vielleicht sogar anti-zionistische - Organisation konzentrierte sich auf die Interessen der jüdischen Gemeinschaft in den Vereinigten Staaten und der jüdischen Diaspora, Israel nicht eingeschlossen, und geriet damit zwangsläufig mit der jüdischen Vertretung in Palästina bzw. mit von Israel aus operierenden Organisationen in Konflikt. Das AJC sprach sich gegen die „israelisch-zionistische Beschlagnahme" der Reparationen und gegen den israelischen Alleinvertretungsanspruch des Weltjudentums aus. Solche Vorstellungen gehen aus einzelnen Dokumenten, die die Bundesrepublik Deutschland betreffen, tatsächlich hervor. ${ }^{59}$ Die Egozentrik bestimmter führender Vertreter war ein weite-

59 Eban an Goldmann vom 9. 3. 1951, Vgl. z. B. ISA, 344/17: „Israel ist der einzige Staat, der im Namen des jüdischen Volkes sprechen kann" oder: DOKUMENTE zUM ABKOMMEN zwischen dem Staat Israel und der Bundesrepublik Deutschland (unterzeichnet am 10. September 1952 in Luxemburg), Jerusalem 1953, Dokument Nr. 5, 25; israelische Note vom 12. März 1951 an die vier Besatzungsmächte betr. Reparationen: „The state of Israel 
rer Faktor der Spannungen und des Machtkampfs zwischen den einzelnen Organisationen.

Deutsch-jüdische Organisationen wurden von anderen jüdischen Organisationen und von Israel als potentielle Bedrohung empfunden. Man befürchtete, Juden deutscher Herkunft könnten sich unter Umgehung einer einheitlichen jüdischen Position für separate Verhandlungen mit der westdeutschen Regierung entscheiden und der deutschen Seite damit die Chance bieten, verschiedene Teile der jüdischen Gemeinschaft gegeneinander auszuspielen. Klagen deutscher Juden, daß jüdische Weltorganisationen einerseits ihr Eigentum beanspruchten, sich andererseits aber zu wenig um die Bedürftigen dieser Gemeinde kümmerten, wurden als Zeichen einer solchen Tendenz interpretiert..$^{60}$ Latent spielte zudem in deutsch-jüdischen Kreisen der im eigenen Selbstverständnis als „deutsche Bürger mosaischen Glaubens" verwurzelte Verdacht mit, von den in großen jüdischen Organisationen besonders stark vertretenen "Ostjuden" hintergangen zu werden. ${ }^{61}$ Ein weiterer Stein des Anstoßes war der Führungsanspruch der zionistischen Organisationen beim Einfordern von Entschädigungszahlungen. Unter den deutsch-jüdischen Organisationen bemühte sich die Axis Victims League im Alleingang besonders heftig um Bonns Wohlwollen.

$\mathrm{Zu}$ weiteren auf eigene Faust operierenden Organisationen zählte der CPRIJG unter der Führung des ehemaligen Chefrabbiners Leo Baeck, eine äußerst verbitterte wie umstrittene Persönlichkeit, sowie die American Association of Jews from Central Europe. Am meisten Spannungen im jüdischen Lager erzeugte der Zentralrat der Juden in Deutschland (ZJD), da er Juden vertrat, die nach dem Krieg in Deutschland lebten, und deshalb besonders daran interessiert war, mit der Bundesregierung in ein gutes Verhältnis zu treten. Auch der Umstand, daß führende Persönlichkeiten des ZJD, wie etwa Hendrik van Dam und Norbert Wollheim, die Anliegen des Zionismus unterstützten, konnte die Befürchtungen gegenüber dieser Organisation nicht zerstreuen. Andere jüdische Organisationen versuchten den ZJD von eigenmächtigem Handeln abzuhalten. Der Umstand, daß sich auch nach dem Krieg noch Juden in Deutschland aufhielten, führte zum ideologischen Konflikt: Vor allem die zionistischen Organisationen und der Staat Israel forderten die restlose jüdische Emigration nach Israel. Ende 1949 schloß die JAFP ihre Emigrationsbüros in Deutschland, ein Schritt, der als Ausgrenzung der in Deutschland gebliebenen Juden aus der jüdischen Weltgemeinschaft interpretiert wurde. Der Jüdische Weltkongreß vertrat dagegen eine konziliantere Haltung und nahm den ZJD als Mitgliedsorganisation auf.62 Dem Druck der zionistischen Organisationen widersetzten sich auch ehemalige, zumeist aus Osteuropa stammende, jüdische DPs, die trotz Spannungen mit Juden deutscher Herkunft

is the appropriate claimant of such reparations, not only because it has borne the major responsibility for salvaging the remnants of European Jewry, but also because it is the only recognized collective entity in which rights adhering to the Jewish people as a whole can find political expression."

60 LBI, S 41/1, M2/1/A, Box 3, Council, IRSO M(a) April 1952 - 30 June 1954.

61 Vermerk Herbert Blankenhorns vom 11. 5. 1954, CZA, Z 6/1622.

62 SHAFIR, Der Jüdische Weltkongreß, S. 218-220. 
Teil der neuen jüdischen Gemeinschaft in Deutschland waren. Auch sie nahmen eine ablehnende Haltung gegenüber dem Zionismus ein und drohten damit, im Alleingang mit der Bundesregierung zu verhandeln.

Insgesamt blieben die jüdischen Organisationen der überkommenen Vorstellung der "Querelles juives" treu. Die rivalisierenden jüdischen Gruppen waren sich jedoch zumindest darin einig, daß Deutschland gegenüber den jüdischen NSOpfern eine große Schuld auf sich geladen habe. Dieser Gedanke führte - zum ersten $\mathrm{Mal}$ in der jüdischen Geschichte - zur Gründung eines gemeinsamen jüdischen Gremiums, auf das wir weiter unten im Text zurückkommen werden.

Doch wie stellte sich die deutsche Seite zur Reparationsproblematik? Die Politik der Bundesrepublik Deutschland gegenüber den Juden hing in jenen Jahren, wie bereits erwähnt, weitgehend vom drängenden Einfluß von außen $a b$, eine Rolle, die Hochkommissar John McCloy gegenüber Bundeskanzler Konrad Adenauer und Bundespräsident Theodor Heuss wahrnahm. Das offizielle Deutschland war weniger daran interessiert, das Verhältnis zu den Juden zu verbessern, als vielmehr die jüdischen (und nichtjüdischen) DPs, die „unerwünschten Ausländer ${ }^{4}$, loszuwerden. ${ }^{63}$ So sind die anfänglichen Kontakte zwischen JAFP-Delegierten und deutschen Regierungsvertretern möglicherweise auf den gemeinsamen Wunsch zurückzuführen, Flüchtlinge nach Israel abzuschieben. Die ersten deutsch-jüdischen Kontakte nach dem Krieg wurden auf jüdischer Seite von der JAFP geknüpft. Ihre Aktivitäten konzentrierten sich zunächst auf Gemeinde- und Länderebene, weiteten sich später aber auch auf die Bundesebene aus.

Deutsche Instanzen waren den Anliegen der Juden nicht besonders wohlgesinnt, was sowohl allgemeine jüdische Kritik an der Restitutions- und Reparationspolitik als auch spezifische Proteste gegen einzelne Zwischenfälle mit deutschen Behördenvertretern hervorrief. Es kam zu Spannungen während der Verhandlungen über die Restitutions- und Reparationspolitik und Auseinandersetzungen über die Umsetzung der bestehenden Restitutions- und Entschädigungsgesetze. Der Hochkommissar sah sich deshalb wiederholt veranlaßt, bei den Ministerpräsidenten der Länder auf eine flexiblere Haltung und ein Ende der bürokratischen Blockierungen hinzuwirken. ${ }^{64}$ Es gab zwar auch gutgesinnte Beamte, mit deren Hilfe anstehende Probleme rasch gelöst werden konnten, doch die auftretenden Schwierigkeiten legen den Schluß nahe, daß der deutsche Beamtenapparat keine Lehre aus dem Holocaust gezogen hatte und weiter den alten antijüdischen Vorurteilen verhaftet blieb. Der Behördenalltag schien zum Status quo

63 Frankfurter RundsChaU vom 23. 6. 1950; vgl.: National Military Establishment, Dept. of the Army, Army releases final Haber report vom 7. 2. 1949, AJA, WJC, U-243; Livne an das Dept. of Economy vom 23. 10. 1949; The Displaced Persons Problem. A Collection of Recent Official Statements; Ausarbeitung von Rabbi Philip S. Bernstein "Status of Jewish Displaced Persons“ vom 20. 6. 1947; JAFP, U.S. Zone Germany, Report of Activities, 1946-1948, vom Februar 1949, ISA 531/6.

64 Bericht von Georg Landauer, über die Arbeit des Bureau for Recovering of Jewish Property in Germany vom 5.4. 1950; McCloy an die Ministerpräsidenten der Länder, 23. Januar 1950, CZA, Z 6/787; Benjamin B. Ferencz an Eli Rock vom 9. 11. 1950, AJDC, Dokument Nr. 4264; Bericht von Benjamin B. Buttenwieser, S. 178, Columbia Oral History Project 1979. 
ante zurückgekehrt zu sein. Anstatt generell eine flexiblere Linie zu beschließen, wurde die Entscheidung offener Fragen einzelnen Beamten überlassen. Zudem setzte sich die neue Verwaltung vorwiegend aus Beamten zusammen, deren $\mathrm{Ge}-$ dankengut noch von NS-Ideologie durchsetzt war. Führende Politiker, wie etwa Justizminister Thomas Dehler (FDP) sowie Finanzminister Fritz Schäffer (CSU), richteten mit unbedachten Äußerungen und einer bürokratischen, formalistischen Haltung ungehindert irreparablen Schaden an. Die unteren Chargen ihrer Ministerien taten es ihnen gleich. Manche Politiker der regierenden Koalition legten den Juden und ihren Ansprüchen gegenüber eine Haltung an den Tag, die bestenfalls als gleichgültig zu bezeichnen ist. Unter diesen Umständen war es ein leichtes, die Entschädigungszahlungen endlos zu verzögern.

Aus der deutschen Bevölkerung waren noch unfreundlichere Töne zu vernehmen. Aufgeschreckt durch das Ausmaß der Restitutionsforderungen, bezeichneten sich die neuen Besitzer "arisierten“ jüdischen Eigentums als Opfer „habgieriger jüdischer Bittsteller“. Sie gründeten Organisationen und führten eine Öffentlichkeitskampagne durch, die ein positives Echo fand. Es bestand die Gefahr, daß diese Gruppierungen zu einer ernstzunehmenden politischen Kraft heranwachsen würden. 65

Bundespräsident Heuss setzte sich derweil an vorderster Front für die Versöhnung zwischen Deutschen und Juden ein. Der ehemalige Vorsitzende der FDP prägte im Dezember 1949 in einer Rede in Wiesbaden den Begriff „Kollektivscham" und forderte die Deutschen dazu auf, sich zu dem Unrecht an den Juden zu bekennen. ${ }^{66}$ Dabei wurde er von Geistlichen, Journalisten und Politikern unterstützt. Auch hochrangige Beamte im Kanzleramt und in der Dienststelle für Auswärtige Angelegenheiten (dem späteren Auswärtigen Amt), darunter Walter Hallstein, Herbert Blankenhorn und Hans Globke, unternahmen größte Anstrengungen in diese Richtung. Ob sie aus schlechtem Gewissen handelten, um sich ein Alibi zu verschaffen - Blankenhorn und besonders Globke galten als belastet -, oder aber aus Pflichteifer, Karrieregründen, um des guten Rufes willen oder schlicht aus menschlichem Anstand: Diese drei Staatsdiener sind gute Beispiele für den unermüdlichen Eifer, den einzelne an den Tag legten, der jüdischen Seite mehr als nur entgegenzukommen. Ehemalige Nationalsozialisten und Mitläufer wandelten sich zu Fürsprechern der Juden. Vor allem in mündlichen Zeugnissen und in der damaligen Presse wurde zuweilen die Ansicht geäußert, daß sich eine deutsche Regierung aus Mitgliedern mit unbelasteter Vergangenheit den Juden und dem Staat Israel gegenüber weniger entgegenkommend zeigen würde.

Dem widersprach jedoch, daß sich gerade die größte Oppositionspartei, die

65 Association for Loyal Restitution, Baden-Baden, Minutes of Meeting held in Kreuznach vom 13. 11. 1949; Eli Rock an Bookstein and Leavitt vom 13.6. 1950, AJDC, Nr. 4264; Ferencz an Jacobsen vom 3. 1. 1950, YIVO, AJC, RG 347, GEN-10, Box 291; Association for Loyal Restitution, Brief Report to our Members, 8. 2. 1951, CZA, A 140/463; KASSELER POST vom 17. 4. 1951; FRANKFURTER RUNDSCHAU vom 30. 4. 1951.

66 Bericht von Norbert Wollheim über das Zusammentreffen mit dem Bundespräsidenten Theodor Heuss am 19.1. und 20. 2. 1950, CZA, A 410/58; vgl. die Rede von Heuss vom Dezember 1949, in der er den Begriff „Kollektivscham “ verwendete, abgedruckt in: THEODOR HEUSS. Politiker und Publizist, S. 381-386. 
SPD, die sich den Nationalsozialisten entgegengestellt hatte, als der jüdischen Seite freundlich gesinnt zeigte. SPD-Spitzenpolitiker wie Kurt Schumacher und Carlo Schmid traten konsequent für die Erfüllung der jüdischen Forderungen ein. ${ }^{67} \mathrm{Die}$ Sozialdemokraten und der ihnen nahestehende Deutsche Gewerkschaftsbund (DGB) spielten eine zentrale Rolle bei der Durchsetzung dieser Ansprüche. Vorstöße der Bundesregierung in Richtung Versöhnung mit den Juden wurden von den Sozialdemokraten als führender Oppositionspartei stets mitgetragen, obwohl es auch in dieser Partei, selbst unter Spitzenvertretern und Abgeordneten, schwarze Schafe gab. Auch in der SPD wogen politisches Kalkül und wahlstrategische Überlegungen manchmal schwerer als moralische Grundsätze. Die Lösung sozialer Probleme ehemaliger Kriegsgefangener, verwundeter Soldaten und Zivilisten sowie Vertriebener schien oft drängender als die Ansprüche der Juden. Insgesamt überwog jedoch bei den SPD-Vertretern die positive Einstellung. Jedenfalls spielte die Partei eine Schlüsselrolle bei der Gesetzgebung in der Restitutionsfrage und ihrer Umsetzung. Zusammenfassend läßt sich sagen, daß die Wiedergutmachungsgegner zwar zahlreicher, aber den Befürwortern gegenüber - vor allem in Schlüsselpositionen - dennoch unterlegen waren.

Eine weitere wichtige Rolle spielten die Entwicklungen im internationalen Umfeld. Zur Zeit der Gründung des Staates Israel und der Bundesrepublik Deutschland war der Kalte Krieg schon in vollem Gange und entwickelte sich bald zum offenen Konflikt im Koreakrieg. Deutschlands Bedeutung war im Steigen begriffen und beide Blöcke strebten danach, die öffentliche Meinung in Deutschland für sich zu gewinnen. Die Gründung der Bundesrepublik bezweckte die Errichtung eines Bollwerks des Westens. Die Bevölkerung galt es davon zu überzeugen, daß eine demokratische Staatsform im westlichen Stil ein nicht zu hoher Preis für die ausbleibende Wiedervereinigung Deutschlands sei, zumal doch der vom Westen gebotene Lebensstandard höher und mit weniger Opfern verbunden sein würde als alles, was die Sowjetunion versprechen bzw. tatsächlich würde bieten können. Hieraus ergab sich die Notwendigkeit des wirtschaftlichen Wiederaufbaus Westdeutschlands durch kräftige Kapitalspritzen und andere materielle Hilfe. Um die zweckgemäße Verwendung dieser Gelder zu garantieren, war die deutsche Wirtschaft einer strengen alliierten Kontrolle unterworfen. Diese förderte die Ausfuhr und blockierte den Geldtransfer - in Form von Devisen oder unbezahlten Warenlieferungen - ins Ausland. Die materiellen Forderungen jüdischer Organisationen und Israels ließen sich mit dieser Politik der westlichen Besatzungsmächte nicht vereinbaren. Selbst die Ausfuhr kleinerer, nach alliierten Gesetzen bereits genehmigter Beträge, war fast unmöglich. Zur Freigabe bestimmte Beträge wurden auf sogenannte Sperrmarkkonten überwiesen, über die nur innerhalb der Bundesrepublik verfügt werden konnte. „Um den Geldbeutel des amerikanischen Steuerzahlers zu schonen", verhinderten die amerikanischen Behörden ungedeckte Exporte aus Westdeutschland.68 Der amerikanische Außenminister Dean Acheson verfolgte die wirtschaftliche Erholung des besiegten Feindes mit besonderer Auf-

67 SHAFIR, Ha yad ha-musheteth.

68 Ausarbeitung „The Problem of Bulk Settlement with Germany“ vom 30. 3. 1950, YIVO, AJC, RG 347, GEN-10, Box 276. 
merksamkeit. Die Entschädignungsansprüche des jüdischen Staates kamen ihm dabei nicht gelegen. ${ }^{69}$ Israelischen Wünschen kamen weder er noch seine Mitarbeiter entgegen, vom britischen und französischen Hochkommissar ganz zu schweigen. Die Reparationsfrage war in eine Sackgasse geraten.

Eine Ausnahme bildete der amerikanische Hochkommissar John McCloy. Mit weitreichenden Befugnissen ausgestattet und weitgehend unabhängig handelnd, genoß er bei der Umsetzung von Anordnungen aus Washington einigen Spielraum. Seine Stimme fand nicht nur Gehör im Weißen Haus, im Außenministerium und im Pentagon, sondern auch im Kongreß. In Deutschland war er deshalb bis 1952 der unumstrittene Boß. Die Politik der wirtschaftlichen Förderung der Bundesrepublik verbunden mit der Devise des sparsamen Umgangs mit amerikanischen Steuergeldern wurde auch von McCloy mitgetragen. Sein Ziel war es, Westdeutschland ins westliche Lager zu führen. Um dies zu erreichen, förderte er die Versöhnung Deutschlands mit seinen Gegnern, allen voran Frankreich. Die Regelung der offenen Fragen zwischen den zwei traditionellen Kontrahenten wurde als bedeutender Schritt in diese Richtung erachtet. ${ }^{70}$

Eine ähnliche Linie verfolgte McCloy offenbar auch gegenüber einem weniger mächtigen Antagonisten Deutschlands, dem jüdischen Volk. Die Juden und der Staat Israel konnten weder Macht ausspielen noch war ihnen, im Gegensatz zu Frankreich, ein internationales Vetorecht gegeben. Ihre einzige Stärke, von der sich eine gewisse Macht ableiten ließ, war ein moralisches Recht. Als Opfer des Holocaust besaß das jüdische Volk einen gewissen Einfluß auf das Gewissen der Weltöffentlichkeit. Das war sein einziger, wenn auch nicht zu unterschätzender Trumpf. McCloy hat diesen Umstand zweifellos erkannt. Ähnliche Versöhnungsschritte wie gegenüber Frankreich, allenfalls in geringerem Umfang, waren also auch gegenüber Israel erforderlich. Während es bei Frankreich vor allem um militärische Sicherheit ging, legte Israel Wert auf materielle Entschädigung. Wenn es also gelang, diese Wünsche zu befriedigen, war das Resultat bei beiden Staaten ein ähnliches: Ihr Veto gegen die Wiederaufnahme der Bundesrepublik Deutschland in die "Völkerfamilie“" würde dahinfallen.

Über McCloys persönliche Beweggründe läßt sich nur spekulieren. Er hatte die deutsche Front kurz vor dem Zusammenbruch des Dritten Reiches besucht und mag dabei Zeuge von NS-Greueln gewesen sein. In seiner bereits erwähnten Heidelberger Rede übte er scharfe Kritik an der NS-Politik gegenüber Juden und Andersdenkenden. Doch auch McCloys Kriegsvergangenheit war nicht frei von dunklen Aspekten. Ihm wurde vorgeworfen, während des Zweiten Weltkrieges antijüdisch gehandelt zu haben und an der Verfolgung von Amerikanern japanischer Herkunft („Nisei“) beteiligt gewesen zu sein. Zudem soll er nach seinem Rücktritt von seinem Posten in Westdeutschland einen antiisraelische Haltung eingenommen haben. ${ }^{71}$ Inwiefern die Vergangenheit seine Handlungen beeinflußte, ist schwer abzuschätzen. McCloy läßt zudem eine unterschiedliche Hal-

69 Interview mit David Ginsburg am 9. 5. 1988, Washington D.C.

70 SCHWARTZ, Atlantik-Brücke, S. 23-24; Interview mit Benjamin B. Ferencz in Long Island, NJ am 28. 5. 1988; Interview mit Saul Kagan in New York, NY am 23. 5. 1988.

71 JELINEK, John McCloy, Jacob Blaustein and the Shilumin, S. 30-31. 
tung gegenüber Juden und Amerikanern japanischen Ursprungs erkennen, was auf folgende Umstände zurückzuführen sein mag: Bevor er in das Verteidigungsministerium eintrat, war McCloy als Firmenanwalt tätig, unter anderem für den jüdischen Großkaufmann und Ölmagnaten Jacob Blaustein, mit dem er sich später befreundete. Diese Freundschaft sollte sich für Blaustein als äußerst wertvoll erweisen. Als McCloy zum amerikanischen Hochkommissar in Deutschland ernannt wurde, wurde Blaustein bei ihm wiederholt mit jüdischen Anliegen vorstellig. Der Beitrag von Blaustein und einiger seiner Helfer war schließlich entscheidend für den Erfolg der Anstrengungen der jüdischen Seite im Hinblick auf deutsche Entschädigung. Es gelang, das alliierte Veto zu überwinden.

\section{Reparationen für die jüdische Gemeinschaft?}

Von den drei Arten von Retribution - Entschädigung, Rückerstattung und Reparationen - stieß letztere auf den größten Widerstand. Der Erlangung von Reparationen wurde deshalb am meisten Aufmerksamkeit geschenkt, da sie, so hoffte man, auch den anderen Formen der Wiedergutmachung den Weg ebnen würden.

Adenauers Initiative vom November 1949 stand unter keinem günstigen Stern. Im Bestreben, die Sache am Leben zu erhalten, legte Karl Marx am 24. des Monats in Israel einen Plan vor, wonach Israel in einem Zeitraum von 12 bis $15 \mathrm{Jahren}$ eine jährliche Güterhilfe im Wert von 200-500 Millionen Mark erhalten solle; doch seine Ideen wurden mit Skepsis aufgenommen.72 Marx genoß, wie erwähnt, einen zweifelhaften Ruf. Er war unter anderem Opfer von Auseinandersetzungen innerhalb der jüdischen Gemeinschaft in Deutschland, an denen er selber nicht ganz unschuldig war. Er beschuldigte den hochangesehenen Norbert Wollheim, seinen Vorschlag zu diskreditieren, als dieser nach einem Gespräch mit Bundespräsident Heuss nach Israel flog. ${ }^{73}$ Marx behauptete später, der Vizekanzler und Minister für wirtschaftliche Zusammenarbeit, Franz Blücher, habe ihm im persönlichen Gespräch die Lieferung von Waren an Israel in Aussicht gestellt und streng geheime Verhandlungen mit israelischen Experten vorgeschlagen. Diesen und einen zweiten, ähnlichen Plan unterbreitete Marx israelischen Regierungsvertretern bei einem weiteren Besuch in Israel. Die Israelis nannten ihn daraufhin einen „Phantasten ". ${ }^{74}$ Man darf annehmen, daß gewisse deutsche Wirtschaftskreise im Rahmen der Suche nach neuen Märkten Interesse für Israels Position im Nahen Osten bekundeten und hofften, durch Wirtschaftshilfe an Israel Arbeitsplätze in der Bundesrepublik zu schaffen. ${ }^{75} \mathrm{Zwar}$ machte man sich in Israel damals durchaus

72 Memorandum von 1953 und Denkschrift vom 26. 7. 1954, Karl Marx Nachlaß; vgl. Shinnar an Ilsar vom 8. 2. 1955, ISA, 590/9.

73 Memorandum von 1953, Karl Marx Nachlaß; Landauer an Kreutzberger vom 17. 2. 1950, CZA, S 35/94.

74 Ebd.; Blüchers Dokumente im BArch, N 1080, enthalten keinen Hinweis auf ein solches Angebot. Blücher unterhielt Kontakt mit Marx und befolgte gelegentlich seinen Rat.

75 Vortrag Arnold Sywotteks "German-Israeli Economic Relations During the Beginning of Contacts between the Two Nations“, gehalten am 20. 3. 1986 an der Ben-Gurion-Universität zu Beer Sheva, BGA, Oral History Division; Informationsübersicht 2/49, Lübeck 
Gedanken über die Reparationsfrage, doch für einen konkreten Vorstoß war das Mißtrauen gegenüber Deutschland noch zu groß.

Israelische Vertreter in Deutschland, darunter Konsul Livneh und JAFP-Delegierte, drängten bei ihren Vorgesetzten darauf, die deutschen Vorstöße ernst zu nehmen. In einem Schreiben an Sharett und Kaplan vom 15. März 1950 warnte Landauer davor, "den guten Willen Adenauers, des einzigen möglichen Verbündeten in Deutschland, zu verspielen" ${ }^{76}$ Nachdem der Kanzler in israelischen Kreisen mehrfach kritisiert wurde, faßte Landauer Mut, ihn - zum ersten Mal in der offiziellen israelischen Korrespondenz - als möglichen Partner zu bezeichnen. Gleichzeitig begann er, die Idee direkter Gespräche mit der deutschen Regierung zu propagieren. Der JAFP-Delegierte in Deutschland, Max Kreutzberger, machte den Vorschlag, Adenauers Geste zu akzeptieren und mit Blankenhorn und dem Adenauer nahestehenden Bankier Robert Pferdmenges das Gespräch zu suchen.77 Am 1. November 1949 eröffnete die JAFP ein Büro in München. Kreutzberger beschäftigte sich mit wirtschaftlichen und rechtlichen Aufgaben. Am 29. Januar 1950 autorisierte die JAFP-Exekutive direkte Gespräche mit deutschen Regierungsvertretern. Auf der Tagesordnung stand unter anderem "die Erlangung voller Entschädigung für den Schaden, den die deutsche Nation jüdischen Personen bzw. dem jüdischen Volk zugefügt hat “ ${ }^{78}$ Darauf erhöhte die Exekutive den Rang des Büros in München und ermächtigte es zu Verhandlungen über Entschädigung sowie zum Empfang verschiedener Entschädigungsleistungen. Das Büro wurde zudem angewiesen, eng mit dem (israelischen) Regierungsausschuß für den Transfer von Entschädigungsleistungen zusammenzuarbeiten, der im Oktober 1949 ins Leben gerufen wurde.

Am 15. Februar $1950 \mathrm{kam}$ das israelische Kabinett auf die deutschen Angelegenheiten zu sprechen und übertrug dem genannten Ausschuß die Behandlung sämtlicher finanzieller Fragen in bezug auf Deutschland. Zum Vorsitzenden wurde der aus Deutschland stammende Peretz (Fritz) Naphtali, Wirtschaftsexperte der Mapai und ehemaliges Mitglied der SPD, gewählt. Am 23. Februar 1950 verfaßte Landauer eine Denkschrift mit dem Titel „Zu verhandelnde Fragen mit der deutschen Regierung bzw. den deutschen Regierungen". ${ }^{79}$ Ein Gremium von zwei Ministern und vier hohen Beamten bzw. Funktionären der israelischen Regierung und der JAFP diskutierte das Papier und beschloß, den in deutschen Angelegenheiten erfahrenen Direktor der Zollbehörde, Kurt Mendelssohn, nach Deutschland zu entsenden. Zum ersten Mal seit der Gründung des Staates Israel wurde ein Regierungsvertreter zu direkten Gesprächen mit der Regierung der Bundesrepublik ermächtigt. ${ }^{80}$ Mendelssohn hatte den Auftrag, die Schwierigkei-

vom 27. 10. 1949, YVA, 0-70/13 (72/38); Kreutzberger an Goldmann vom 9. 2. 1950, $\mathrm{CZA}, \mathrm{Z}$ 6/387; H. Kox an den Minister über den Abteilungsleiter Staatssekretär vom 20. 6. 1950, PA, 210-01/35, Bd. 2, Abt. II/IIa; HAARETZ (Tel Aviv) vom 4. 6. 1950.

76 Landauer an Sharett and Kaplan vom 15. 3. 1950, CZA, S 35/185.

77 Kreutzberger an Goldmann vom 9. 2. 1950, CZA, Z 6/387.

78 Ausarbeitung „Decisions concerning Germany“ vom 29. 1. 1950, CZA, A 140/58.

79 Mit Datum vom 22. 3. 1950, CZA, S 35/18.

80 Protokoll der Besprechung mit Vertretern der Regierung und der Jewish Agency vom 2. 5. 1950, CZA, A 140/58. 
ten der JRSO bei der Sicherstellung des erbenlosen Eigentums und die Probleme beim Transfer von Entschädigungsgeldern zu erörtern. Weitere Tagesordnungspunkte betrafen das schleppende Tempo der Entschädigungszahlungen, die Schätzung des (jüdischen) Eigentums durch deutsche Behörden sowie den Einkauf. Mendelssohns Mission markierte eine Wende im israelischen Denken und bedeutete gleichzeitig den Bruch des inoffiziellen Boykotts gegen Deutschland. Mendelssohn war zwar nicht der erste israelische Emissär in Deutschland, doch seine Vorgänger waren ohne offizielle Erlaubnis zu Gesprächen nach Deutschland gereist und dabei gescheitert. Dieses Mal schienen Marx' Aktivitäten Früchte zu tragen. Sharett, Kaplan und Naphtali genehmigten die Entscheidung des Ausschusses und sorgten für die Zustimmung der Regierung.

Doch Mendelssohns Mission war nicht nach dem Geschmack von Landauer und der JAFP-Gesandtschaft in München, die ihr jede Hilfe versagten. JAFP-Delegierte wiesen auf den Schaden hin, den Mendelssohn ihrer Ansicht nach anrichtete, und bis zu einem gewissen Grad sollten sie recht behalten, wie noch zu zeigen sein wird. Andererseits standen Mendelssohn als Regierungsbeauftragtem Türen offen, die den JAFP-Delegierten verschlossen waren. Das AJC bezichtigte die israelische Regierung im Zusammenhang mit der Mendelssohnschen Mission der Einmischung in laufende Verhandlungen der JRSO. Dahinter stand natürlich die Befürchtung des AJC, die Israelis könnten versuchen, den Alleinvertretungsanspruch des jüdischen Volkes durchzusetzen. Ben Gurion hätte in weiser Voraussicht mehrmals erklärt, er betrachte den Staat Israel nicht als Vertreter des Weltjudentums, hieß es dazu warnend in AJC-Kreisen. ${ }^{81}$ Goldmann gelang es schließlich, das AJC mit der - unwahren - Erklärung zu beschwichtigen, Israel gehe es nur um das Eigentum seiner Bürger deutscher Herkunft. ${ }^{82}$ Mendelssohns offizielles Mandat beschränkte sich zwar tatsächlich auf das Einholen von Informationen über die Entschädigung für in Israel lebende Juden deutscher Herkunft, doch im Endeffekt bezweckten seine Sondierungen einen umfassenden Ausgleich mit der Regierung der Bundesrepublik Deutschland. Er hielt sich mit Unterbrechungen drei Monate in der Bundesrepublik auf. In dieser Zeit führte er Gespräche mit den Bundesministern Blücher und Schäffer sowie mit hohen und mittleren Beamten. Mit Wirtschaftsvertretern erörterte er mögliche Handelsbeziehungen.

Mendelssohn kam bei seinen Gesprächen in Deutschland sowohl jüdischen Organisationen als auch seinen Gastgebern in die Quere, möglicherweise auch weil er sich der Komplexität der anstehenden Probleme nicht genügend bewußt war. Der israelische Emissär mischte sich in Angelegenheiten ein, die gleichzeitig auch von der JRSO, der URO, dem israelischen Büro für Forderungen gegenüber Deutschland (Miltam) und der JAFP-Abteilung für die Rückführung des Eigentums deutscher Juden (DRPGJ) behandelt wurden. Sein offizieller Status stiftete zudem Verwirrung bei den Gastgebern, und in der deutschen Presse wurde er fälschlicherweise als Vizefinanzminister bezeichnet. Mendelssohns Interventionen verschafften deutschen Regierungsvertretern Gelegenheit, Entschädigungs-

81 Proskauer an Goldmann vom 5. 4. 1950, CZA, Z 6/387; Telegramm von Proskauer an Goldmann vom 5. 4. 1950, YIVO, AJC, RG-1, EXO-16.

82 Goldmann an Proskauer vom 19. 4. 1950, CZA, Z 6/387. 
maßnahmen zu verzögern und die jüdischen Organisationen gegeneinander auszuspielen. Andererseits war es das Verdienst von Mendelssohns Mission, die deutsche Seite zu einer ernsthaften Erwägung der Entschädigungsfrage zu bewegen. Wie Landauer berichtet, hat Mendelssohn der deutschen Seite eigenmächtig den Vorschlag gemacht, Israel Waren im Wert von 100 Millionen Mark als Anzahlung einer sofortigen Entschädigung im Umfang von 250 Millionen Mark zu liefern. Sodann habe er mit den Ministern Blücher und Schäffer die gesamten israelischen Ansprüche und die Möglichkeiten einer globalen Lösung erörtert. ${ }^{83}$ Wenn auch die Vorteile einer raschen Regelung dieser Frage auf deutscher Seite durchaus erkannt wurden, kam das Bundesministerium der Finanzen zu dem Schluß, daß man nicht über die dafür nötigen Mittel verfüge, und am 9. Juni 1950 beschloß die Bundesregierung, gestützt auf Schäffers Vorschlag, die Angelegenheit vorläufig zurückzustellen. ${ }^{84}$ "Fehlende Mittel $^{\text {“ }}$ waren offensichtlich nicht der einzige Grund: Die Gespräche hatten bereits eine gewisse Spannung in der westdeutschen Wirtschaft und speziell auch auf dem bundesdeutschen Immobilienmarkt erzeugt. ${ }^{85}$ Zudem reagierte die Öffentlichkeit mit großem Unwillen auf die jüdischen Forderungen, was die Politiker dazu bewog, vorläufig keine Entscheidung zu treffen.

Eine weitere schwere Belastung für die Bundesrepublik war die Entschädigungsfrage. In den Wirtschaftsministerien und in der Dienststelle für Auswärtige Angelegenheiten war man bestrebt, die Frage auf einmal zu erledigen, und Bundesminister Schäffer stellte Nachforschungen an, welcher Betrag dafür aufzuwenden wäre. Für eine große Summe war die Zustimmung des Bundestags erforderlich. Ein längeres Schriftstück des Bundesministeriums der Finanzen erklärte die wirtschaftlichen und politischen Vorteile eines Abkommens mit Israel, erwähnte Mendelssohn und endete mit folgendem bemerkenswerten Satz: „Bei allen diesen Überlegungen sollte beachtet werden, daß neben der Verbesserung des Verhältnisses zwischen Deutschland und Israel der politische und moralische Kredit der Bundesrepublik wächst. Auch das muß sich über kurz oder lang wirtschaftlich auswirken." 86

Die Bonner Regierung war sich des moralischen Vorteils einer Anerkennung des Staates Israels sehr wohl bewußt. Man war bestrebt, Israel zu der Aufnahme von offiziellen Beziehungen mit der Bundesrepublik Deutschland zu bewegen. Im Schlußbericht über seine Gespräche unterstrich Mendelssohn am 7. Juli 1950, daß er als offizieller Vertreter des Staates Israel auf deutscher Seite besonderes Interesse geweckt habe: „Sie sind daran interessiert, die Entschädigungsforderungen auf einer kollektiven Basis zu formalisieren, wie es zwischen Staaten üblich ist.“ Jeder Ansatz zur Aufnahme von Kontakten auf staatlicher Ebene sei auf deutsches

${ }^{83}$ Zusammenfassung eines Gesprächs mit dem Finanzminister Herr Dr. Schäffer im Bundesministerium der Finanzen vom 5.6. und 7.6. 1950, Bonn, CZA, S 35/70.

84 Die Kabinettsprotokolle Der Bundesregierung, Band 3: 1950, 72. Kabinettssitzung, 9. Juni 1950.

85 Bericht von Dr. Mendelsohn vom 17. 7. 1950, ISA, 2482/13.

86 Abt. II/IIa, Herr Kox, an den Herrn Minister mit der Bitte um Kenntnisnahme vom 20. 5. 1950 (in Abschrift), PA, 210-01/35, Bd. 2. 
Interesse gestoßen. ${ }^{87}$ Ein JAFP-Schriftstück faßt die Logik von Mendelssohns Mission wie folgt zusammen: Die Regierung wird sich entweder dazu entschlieBen, eine ständige Vertretung in Deutschland zu eröffnen oder sich definitiv von der Behandlung der Entschädigungsfrage zurückziehen. ${ }^{88}$ Eine Äußerung, aus der der Monopolanspruch der JAFP in dieser Frage deutlich hervorgeht. Andererseits hatte Mendelssohns Mission auf israelischer Seite die Einsicht gefördert, daß die israelischen Forderungen ohne direkte Gespräche zwischen den Regierungen nicht durchzusetzen waren.

In Deutschland war inzwischen ein Streit darüber entbrannt, ob der Bund oder die Länder die Last der Entschädigungen zu tragen hätten. Ein besonderer Ausschuß unter der Leitung des hessischen Finanzministers und Vorsitzenden des Finanzausschusses des Bundesrats, Werner Hilpert, erhielt den Auftrag, Vorschläge zur Finanzierung von Zahlungen im Rahmen einer Entschädigungsvereinbarung auszuarbeiten. Unter den am Ausschuß beteiligten Regierungsvertretern befand sich auch der umstrittene Bayerische Staatskommissar für religiös, rassisch und politisch Verfolgte, Philipp Auerbach. Der Ausschuß sprach sich gegen eine Anzahlung aus, und die zahlreichen Feinde des Wiedergutmachungsexperten Auerbach zögerten nicht, ihm die Verantwortung für die negative Entscheidung zuzuschieben. Nachfolgende Debatten über weitere Möglichkeiten einer globalen Entschädigung verliefen ebenfalls ergebnislos. Sämtliche Vorschläge waren an den Zuständigkeitskonflikten der Länder in der Wiedergutmachungsfrage und an der finanziellen Lage des Bundes gescheitert. ${ }^{89}$ Auerbach, dessen Vergangenheit als umstritten galt, wurde später im Zusammenhang mit einem Korruptionsskandal von der bayerischen Polizei verhaftet und nahm sich unter merkwürdigen Umständen in seiner Haftzelle das Leben. 90

Im Rückblick scheinen Mendelssohns Sondierungen erfolgreicher gewesen zu sein, als es aus seinem eigenen Bericht oder aus der Kritik der JAFP-Delegierten hervorging. Nach der JAFP gelangte auch das israelische Außenministerium zur Einsicht, daß die Entschädigungsfrage ohne direkte Gespräche nicht zu lösen sei. Nun galt es, diese Erkenntnis der politischen Führung weiterzuvermitteln. Die JAFP entwickelt eine Anzahl konstruktiver Lösungsvorschläge, und der auf höchster politischer Stufe aktive Goldmann sowie die täglich mit deutschen Stellen verkehrende DRPGJ entfalteten eine rege Tätigkeit. Goldmann versuchte auf die Entscheidungsträger - McCloy, Adenauer und Heuss - einzuwirken, und suchte den besten Weg, um die Alliierten und die Deutschen zu einer Zusammenarbeit mit der jüdischen Seite zu bewegen. Er bezweifelte den Nutzen einer Friedenskonferenz für die jüdischen Anliegen und warnte, die Deutschen könnten ihr Interesse am

87 Zusammenfassung der Gespräche mit der westdeutschen Regierung betr. Wiedergutmachung für Israelische Staatsbürger und "Haavarah" vom 7. 7. 1950, CZA, S 35/70; vgl.: Gerling an Landauer vom 7.7. 1950, CZA, S 35/70; Livneh an das Außenministerium, Abteilung Wirtschaft, vom 14. 7. 1950, ISA, 2543/1.

88 Gerling an Moses vom 17. 7. 1950, CZA, S 35/70.

89 Ostermann an Tolkowsky vom 10.1.1951, PA, 210-01/35, Bd. 1; vgl. auch: Tolkowsky an Ostermann vom 27. 10. 1950 und Ostermann an Tolkowsky vom 21. 12. 1950, PA, 210 01/ 35, Bd. 1.

90 GosCHLER, Der Fall Philipp Auerbach, S. 77-98. 
Ausgleich mit den Juden verlieren, wenn die „Gegenleistung“, die er dann auch benannte, weiter im unklaren bleibe: Was die jüdische Seite zu bieten habe, sei eine „politische Rehabilitation Deutschlands im Gegenzug für die Lösung der Restitutionsfrage". ${ }^{91}$ Diese Darstellung war nicht neu, doch eine für die jüdische Seite günstige Umsetzung in praktische Politik bedingte die richtigen Partner und den geeigneten Zeitpunkt. Goldmann versuchte deshalb Zeit zu gewinnen. Demgegenüber befürworteten Landauer und seine Anhänger eine Lösung gemäß dem Vorbild der Haavarah. Landauer schlug die Gründung einer übergeordneten jüdischen Restitutionsstelle (Jewish Restitution Center) zur Koordinierung der Arbeit der verschiedenen in Deutschland aktiven jüdischen Organisationen vor. Eine solche Stelle, hoffte Landauer, würde die Rivalität zwischen den einzelnen Organisationen eindämmen und die Gefahr bannen, daß deutsche Stellen kleinere Antragsteller mit kleinen Summen abspeisten. ${ }^{92}$ JAFP-Vertreter schlugen eine globale Lösung für die noch ausstehenden jüdischen Forderungen vor. Landauer nannte die Summe von drei Milliarden Mark, die etwa jenem Betrag entsprach, auf den man sich im Luxemburger Abkommen von 1952 tatsächlich einigte. Als Zahlungsfrist nannte Landauer die Zeitspanne von zwei bis fünf Jahren. Der Transfer nach Israel sollte zudem in Form von Warenlieferungen über eine eigens zu diesem Zweck zu gründende Organisation geschehen, mit Hilfe von Firmen, die sich bereits bei der Haavarah bewährt hatten. Als Kuriosum wäre noch anzufügen, daß man erwog, zu diesem Zweck deutsche Beamte wieder anzustellen, die sich in den dreißiger Jahren mit dieser Angelegenheit beschäftigt hatten.

Landauers Pläne kamen der Realität ziemlich nahe. Während die JRSO auf Länderebene bereits globale Lösungen in die Tat umsetzte, um langwieriges Ringen um Teillösungen zu vermeiden, waren Goldmann, Blücher und andere Bundesstellen immer noch mit dem Entwurf und der Diskussion von Plänen beschäftigt. Die jüdisch-israelisch-deutschen Verhandlungen im Jahre 1952 erreichten schließlich eine globale Lösung auf zwei Ebenen: Erstens hinsichtlich der Ansprüche der sogenannten Claims Conference und zweitens in bezug auf die individuelle Entschädigung für in Israel lebende Opfer des Nationalsozialismus. Der Umstand, daß nicht das Landauer-Programm umgesetzt wurde, kommt einer Ablehnung der JAFP-Schirmherrschaft gleich, obwohl, wie erwähnt, große Teile dieses Programms (nämlich des Haavarah-Programms) im Luxemburg-Abkommen und bei dessen Umsetzung - durch die israelische Regierung und nicht durch die JAFP - zur Geltung kamen. Die Diskussion des Landauer-Plans trug keine Früchte, und der Anspruch des Staates Israel, die Regelung der deutsch-jüdischen Angelegenheiten in seine Hände zu nehmen, bedeutete das Ende seines Auftrags. Doch Landauer gab nicht auf. Im Staate Israel und in dessen Vertreter Livneh erkannte er seine hauptsächlichen Widersacher, und um unabhängig zu bleiben, blieb ihm

91 Minutes of the joint meeting of the sponsoring agencies of JRSO and representatives of the government of Israel vom 2. 5. 1950, AJDC.

92 Ausarbeitung von Georg Landauer „Global Arrangement of Jewish Claims Related to Restitution of Jewish Property and Indemnities in Germany “ vom Juni 1950, CZA, A 140/58; Memorandum von Georg Landauer zur Restitution vom 5. 8. 1950, LBI, B26/1, Nr. 17. 
nichts anderes übrig, als die Vormachtstellung seines Büros gegen sie zu verteidigen.

Bei Meinungsverschiedenheiten zwischen der JAFP und der israelischen Regierung über Strategie und Taktik in deutschen Angelegenheiten schimmerten auf beiden Seiten Machtstreben bzw. der Ehrgeiz durch, die Politik zu diktieren und deren Durchführung in eigener Regie zu gestalten. Landauers Chancen verschlechterten sich aber zusehends, da die JAFP gegenüber dem Staat Israel immer mehr an Boden verlor. Zudem tobte ein erbitterter Machtkampf zwischen den JAFP-Vertretungen in New York und Jerusalem. Auch waren gewisse JAFP-Delegierte in Deutschland offensichtlich nicht weitsichtig genug, um sich die Unterstützung anderer in Deutschland aktiver jüdischer Organisationen zu sichern. Im Gegenteil. Im Kampf um Zuständigkeiten standen sie dem AJC, dem Jüdischen Weltkongreß, dem ZJD und weiteren Organisationen gegenüber, konnten also nicht mit ihnen als potentielle Verbündete rechnen. Persönliche Spannungen und Eigenheiten sowie Mentalitätsunterschiede spielten zudem eine nicht zu unterschätzende Rolle. Landauer etwa verwies auf sein ,jeckisches Erbe (Jecke $=$ spött. isr. Bez. für deutscher Jude) mit seiner fehlenden Anpassungsfähigkeit" ${ }^{\text {" }}{ }^{93}$ Sharett bemerkte abschätzig über Kreutzberger, seine Eigenheiten seien schon längst bekannt. ${ }^{94}$ Diese Persönlichkeiten deutsch-jüdischer Herkunft waren in israelischen und amerikanischen Kreisen nicht sonderlich beliebt. Insofern wirkte sich der persönliche Faktor verschärfend auf den Machtkampf zwischen den einzelnen Organisationen aus. Zeitlebens ein überzeugter Zionist, starb Landauer als verbitterter Mann und fand in New York seine letzte Ruhe.

Beamte des israelischen Außenministeriums warfen der DRPGJ vor, sich gegen die jüdischen Reparationsforderungen zu stellen und die diplomatischen Anstrengungen des Staates Israel zu untergraben. Mit ihrer Politik der kleinen Schritte habe sich die DRPGJ der deutschen Regierung zu billig verkauft. Während Israel Milliarden von Dollars fordere, komme die Bundesrepublik Deutschland dank JAFP billig davon. Des weiteren beschuldigten die Vertreter des Außenministeriums die DRPGJ, aus Furcht vor dem Verlust der Vormachtstellung in deutschen Angelegenheiten eigenmächtig zu handeln, Beschlüsse der israelischen Regierung zu mißachten, Information zurückzuhalten und zu versuchen, offizielle israelische Vertretungen bei ihrer Arbeit zu behindern.

Die wachsende Spannung zwischen den israelischen Regierungsbehörden und der JAFP führte am 5. September 1951 zu einer Aussprache auf höchster Ebene. Zu den Teilnehmern zählten Sharett, Kaplan, Eschkol, Goldmann und weitere führende Politiker sowie JAFP-Vertreter und Beamte des Außenministeriums. Das emotionsgeladene Treffen endete mit der Wahl eines Ausschusses, dem die Aufgabe zufiel, die Verantwortlichkeiten der israelischen Regierung und der JAFP festzulegen. Shinnar und Landauer verfaßten ein Schlußdokument mit folgenden fünf Punkten: 1. Die israelischen Ansprüche haben Vorrang vor allen anderen Ansprüchen, 2. Nur Regierungsvertreter sind zu Verhandlungen über deutsche Reparationen ermächtigt, 3. Die Regierung unterstützt die Durchsetzung indivi-

93 Landauer an Locker vom 12. 7. 1951, CZA, S 35/39.

94 Sharett an Avner vom 6. 9. 1951, ISA, 2417/3. 
dueller Ansprüche, 4. Nichtstaatliche Organisationen sind für die individuelle Entschädigung und Restitution verantwortlich, 5. Wo gemeinsame Interessen und gemeinsame Aufgaben vorliegen, bestimmen die Regierung und die JAFP einvernehmlich die genaue Art der Zusammenarbeit. Die beiden Verfasser unterzeichneten das Abkommen am 14. Oktober 1951.95 Obwohl das Dokument formal eine Aufgabenteilung festlegte, war die JAFP die klare Verliererin. Sie mußte fortan mit einer zweitrangigen Rolle vorlieb nehmen und war der Regierung weitgehend unterstellt. Das kam zwar nicht überraschend, war für die Leitung der JAFP aber dennoch ein harter Schlag: Auch beschwichtigende Erklärungen, daß Landauer auch fortan konsultiert werde und an den Verhandlungen mit der Bundesrepublik Deutschland teilnehme, konnten nicht darüber hinwegtäuschen, daß er nicht mehr $\mathrm{zu}$ den Entscheidungsträgern gehörte. Angesichts der direkten Beteiligung der Regierung an der Behandlung der deutschen Angelegenheiten kam der JAFP nur noch eine untergeordnete, beratende Rolle zu. Und nach der Gründung des weltweiten jüdischen Gremiums (Claims Conference) für Verhandlungen über individuelle Ansprüche büßte die JAFP auch in diesem Bereich ihre Daseinsberechtigung ein. Die DRPGJ, Wegbereiter der Retributionsansprüche gegenüber Deutschland, hatte ausgedient.

Der Staat Israel sprach im Namen seiner Bürger und, ohne es offen auszusprechen, auch im Namen der jüdischen Gemeinschaft außerhalb Israels. Die JAFP stand für die Zionisten, während sich der Jüdische Weltkongreß zur Vertretung der jüdischen Diaspora berufen fühlte. Als nichtstaatliche Organisation konnte sich der Jüdische Weltkongreß in seinen öffentlichen Stellungnahmen zu Schuld und Entschädigungspflicht der Deutschen einen schärferen Ton und eine klarere Sprache als die israelische Regierung erlauben und damit offen aussprechen, was auch viele Israelis, darunter Regierungsvertreter, dachten. Ein Beispiel hierfür ist die Resolution, die der Jüdische Weltkongreß Ende 1949 verabschiedete, in der es unter anderem hieß:

„Die Welt und besonders das jüdische Volk bezweifeln, daß das deutsche Volk das enorme Ausmaß des Verbrechens überhaupt begreift und dafür Buße leisten kann, solange es sich nicht formell und als Kollektiv zur Schuld bekennt [...] Der deutsche Staat und das deutsche Volk müssen sich formell zur Wiedergutmachung des Unrechts bekennen, das dem jüdischen Volk zugefügt wurde, und Reparationen leisten, die es den Überlebenden erlauben, ihre Existenz in Israel oder anderswo in Freiheit und Sicherheit wiederaufzubauen." ${ }^{\text {"96 }}$

Im September 1950 verfaßte der Jüdische Weltkongreß ein weiteres Memorandum zu den jüdischen Forderungen, das er der vom 12. bis 14. September in New York tagenden Außenministerkonferenz übersandte, auf der auch die Rolle der Bundesrepublik innerhalb der westlichen Gemeinschaft zur Debatte stand. ${ }^{97}$ In ihm

95 Shinnar to Landauer vom 18. 10. 1951, incl. Anhänge; Livneh an Shinnar vom 27. 12. 1951, ISA, 534/1.

96 Resolution "Jews and Germans" vom 30. 1. 1950, YIVO, AJC, FAD-1, Germany/West, Box 3.

97 WJC an den Rat der Außenminister der Vereinigten Staaten, Großbritannien und Frankreich vom 12.-14. 9. 1950, Memorandum über jüdische Forderungen, CZA, S 35/39; vgl. SHAFIR, Der Jüdische Weltkongreß, S. 217-218; siehe auch SHAFIR, Ambiguous Relations; TEMPEL, Legenden der Allmacht. 
wurde den kollektiven Ansprüchen erneut Nachdruck verliehen. Auf jeder der folgenden Tagungen kam der Jüdische Weltkongreß sodann auf die Entschädigungsansprüche zurück. Die nächste aufsehenerregende Erklärung wurde fast gleichzeitig mit den israelischen Noten an die Alliierten und möglicherweise zu deren Verstärkung Anfang 1951 veröffentlicht. Die halbamtliche Diplomatische Korrespondenz reagierte auf den jüngsten Vorstoß des Jüdischen Weltkongresses mit Kritik. Bemängelt wurde die Forderung eines Kollektivschuldbekenntnisses und allgemein die feindselige Linie gegenüber Deutschland. ${ }^{98}$ Angesichts der Vorstöße des israelischen Außenministeriums erübrigten sich die Aktionen des Jüdischen Weltkongresses, doch solange sich die Aktivitäten der Israelis auf diskrete diplomatische Kanäle beschränkten, sorgte der Weltkongreß dafür, die jüdischen Ansprüche im öffentlichen Bewußtsein wachzuhalten.

Bevor die israelische Regierung diplomatische Initiativen ergreifen konnte, kam es zu zusätzlichen lokalen, einander teilweise überlappenden Aktionen, die neue Möglichkeiten aufzeigten. Einen solchen Vorstoß wagte der WJC-Vertreter Noah Barou, der fest vom Potential direkter Verhandlungen mit der Bundesrepublik Deutschland überzeugt war. Er führte Gespräche mit Vertretern der Bundesregierung und machte dabei über seinen Geschäftspartner, den deutschen Emigranten Gerhard Lewy, die Bekanntschaft des damaligen Kölner Oberbürgermeisters, Hermann Pünder, der ihm offenbar Zugang zu hohen Vertretern des Bundesministeriums der Finanzen und des Kanzleramts verschaffte. Auf diesem Weg lernte er auch Herbert Blankenhorn kennen, der mit der Familie Lewy freundschaftliche Kontakte unterhielt. ${ }^{99}$ Ein weiterer gemeinsamer Freund Barous und Lewys war Michael A. Thomas (alias Ulrich Holländer), ein britischer Verbindungsoffizier, der als Kontaktmann zwischen dem ehemaligen Kölner Oberbürgermeister Konrad Adenauer und der britischen Besatzungsverwaltung fungiert hatte. $\mathrm{Zu}$ diesem Kreis stießen noch weitere Personen, darunter der Geschäftsmann Joseph (Yossl) Rosensaft, KZ-Überlebender und Vorsitzender der Displaced Persons aus Bergen Belsen, der später beschuldigt wurde, mit Schwarzmarktgeschäften ein Vermögen angehäuft zu haben. ${ }^{100}$ Dem mit Norbert Wollheim vom ZJD befreundeten Rosensaft gelang es bald, freundschaftliche Kontakte mit Ben Gurion, Sharett und Goldmann zu knüpfen. Zudem glückte es ihm, Verbindungen mit einer Reihe von politisch einflußreichen Persönlichkeiten in Bonn herzustellen, so daß er längere Zeit als graue Eminenz der deutsch-jüdischen Verhandlungen galt.

In einem - vermutlich von Barou autorisierten - Schreiben an Pünder vom 26. März 1950 wies Lewy auf die Vorteile hin, die der Bundesrepublik aus einer

98 JÜDISCHER WELTKONGRESS ERGREIFT INITIATIVE. In: AWJD vom 9. 3. 1950; Der JÜDISCHE WELTKONGRESS UND DIE WIEDERGUTMACHUNG. In: Diplomatische Korrespondenz vom 10. 3. 1951.

99 Gerhard Lewy an Pünder vom 26. 3. 1950; Gerhard Lewy an Blankenhorn vom 10.7. 1951, PA, 244-1011/51; Eintrag vom 4. 4. 1950; Pünder an Lewy vom 19. 5. 1950, BArch, N 1351/3; Blankenhorn an Barou vom 12.11. 1951, BArch, N 1351/86; Blankenhorn an Barou vom 18. 7. 1951, IJA, 220.0.

100 Dieser Verdacht wurde von verschiedenen Seiten erhoben. So etwa in einem Brief von Michael A. Thomas an den Autor, 21. 7. 1989. 
entgegenkommenderen Politik gegenüber den Juden erwachsen würden. In diesem Zusammenhang nannte er die Forderungen der jüdischen Seite, darunter eine Erklärung im Bundestag, die eine Verurteilung der NS-Politik sowie eine Verpflichtung zur Zahlung von Entschädigungen und zum Kampf gegen den Antisemitismus enthalten müsse. ${ }^{101}$ Die geforderte Erklärung im Bundestag wurde als eine Vorbedingung für „Friedensverhandlungen“ $z$ wischen führenden Vertretern jüdischer Organisationen und deutschen Regierungsvertretern bezeichnet. Nachdem Lewy Pünder die Hilfe Barous angeboten hatte, traf Blankenhorn am 4. und 28. April 1950 zweimal mit Barou zusammen, der die jüdischen Forderungen bekräftigte. Von Blankenhorn war es bekanntlich nicht weit zu Adenauer. Darüber hinaus hatten Lewy, Barou und ihre - überwiegend nichtjüdischen - deutschen Geschäftspartner Zugang zu höchsten Stellen der westdeutschen Verwaltung und Wirtschaft, darunter Ludwig Erhard, Robert Pferdmenges, Hermann Josef Abs und Vollrath von Maltzan. Ihrer jüdischen Abstammung wegen wurden Lewy und seine Freunde besonders umworben, da Bonn die Juden als wichtige Mittler betrachtete. ${ }^{102}$ Der Kreis um Lewy und Barou erkannte die Gelegenheit zum profitablen Geschäft und tat sein Bestes, sie in die Tat umzusetzen. ${ }^{103} 1952$ und 1953 sah sich die israelische Regierung dann zu drastischen Schritten gezwungen, um sich von dieser Gruppe zu distanzieren, deren Einfluß bis zur Eröffnung der israelischen Mission in Köln im Jahre 1953 anhielt.

Zusammenfassend läßt sich sagen, daß die Annäherung zwischen Israel und der Bundesrepublik bis zu jenem Zeitpunkt mit beträchtlichem persönlichen Nutzen für sämtliche Beteiligten verbunden war. Es steht außer Frage, daß diese europäischen Vertreter des Jüdischen Weltkongresses einen bedeutenden Beitrag zur Knüpfung direkter Kontakte zwischen Bonn und Jerusalem geleistet haben. Adenauer war mit an Sicherheit grenzender Wahrscheinlichkeit von Blankenhorn über die erwähnten Kontakte unterrichtet worden. Doch bei der Herstellung direkter Kontakte mit Israel zog der Kanzler die offiziellen Kanäle vor. Barous Beitrag lag also eher in der Schaffung geeigneter Rahmenbedingungen und des geeigneten psychologischen bzw. politischen Klimas als in der konkreten Anbahnung der ersten direkten Verhandlungen. Seine direkte Linie zu Blankenhorn büßte jedoch auch später nichts von ihrer Wichtigkeit ein - ein Treffen zwischen Goldmann und Blankenhorn kam durch seine Vermittlung zustande -, wenn er auch nicht der einzige Vermittler zwischen jüdischen und deutschen Vertretern war.

Während man in israelischen Regierungskreisen noch hoffte, daß die Deutschen aus eigener Initiative ein neues Kapitel in den deutsch-jüdischen Beziehungen aufschlagen würden oder - noch besser - daß ein solcher Schritt von dritter

101 Lewy an Pünder vom 26. 3. 1950; vgl. auch Lewy an Blankenhorn, 10. 7. 1951, PA, 244$1011 / 51$.

102 Vgl. dazu in einem Schreiben von Lewys Rechtsanwalt Kubutschok an den Finanzminister des Landes Nordrhein-Westfalen vom 4. 3. 1950 (BArch, N 1005, Bd. 630) die starke Hervorhebung des Umstands, daß Lewy und seine Geschäftsfreunde Juden waren.

103 Brief von Michael A. Thomas an den Autor vom 21. 7. 1989, in dem dieser über Gerhard Lewy schrieb, dieser „glaubte an die Wiedergutmachung, doch bei allem Respekt für seine großen Anstrengungen war sie für ihn doch vor allem eine Business-Gelegenheit“. 
Seite erwirkt werden könnte, verfolgte Barou die Idee des direkten Gesprächs. Weder die Botschaften von Marx und Mendelssohn, noch Landauers Bemühungen konnten erreichen, was Barou gelang: Er sprach mit deutschen Vertretern und sagte ihnen, was sie hören wollten. Der erste Schritt zum deutsch-jüdischen Dialog gilt deshalb als Barous Verdienst. 